Article

\title{
Properties of Degradable Polyhydroxyalkanoates (PHAs) Synthesized by a New Strain, Cupriavidus necator IBP/SFU-1, from Various Carbon Sources
}

\author{
Natalia O. Zhila ${ }^{1,2, *}$, Kristina Yu. Sapozhnikova ${ }^{1,2} \mathbb{D}$, Evgeniy G. Kiselev ${ }^{1,2} \mathbb{D}$, Alexander D. Vasiliev ${ }^{1,3} \mathbb{D}^{\mathbb{D}}$,

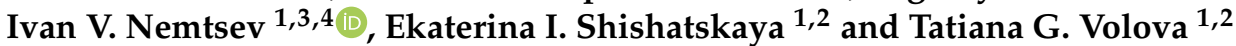 \\ 1 Basic Department of Biotechnology, School of Fundamental Biology and Biotechnology, Siberian Federal \\ University, 79 Svobodnyi Av., 660041 Krasnoyarsk, Russia; kristina.sap@list.ru (K.Y.S.); \\ evgeniygek@gmail.com (E.G.K.); adva@iph.krasn.ru (A.D.V.); ivan_nemtsev@mail.ru (I.V.N.); \\ shishatskaya@inbox.ru (E.I.S.); volova45@mail.ru (T.G.V.) \\ 2 Federal Research Center "Krasnoyarsk Science Center SB RAS", Institute of Biophysics SB RAS, 50/50 \\ Akademgorodok, 660036 Krasnoyarsk, Russia \\ 3 Federal Research Center "Krasnoyarsk Science Center SB RAS", L.V. Kirensky Institute of Physics SB RAS, \\ 50/38 Akademgorodok, 660036 Krasnoyarsk, Russia \\ 4 Federal Research Center "Krasnoyarsk Science Center of the Siberian Branch of the Russian Academy of \\ Sciences", 50 Akademgorodok, 660036 Krasnoyarsk, Russia \\ * Correspondence: nzhila@mail.ru; Tel.: +7-391-290-54-91; Fax: +7-391-243-34-00
}

\section{check for} updates

Citation: Zhila, N.O.; Sapozhnikova, K.Y.; Kiselev, E.G.; Vasiliev, A.D.; Nemtsev, I.V.; Shishatskaya, E.I.; Volova, T.G. Properties of Degradable Polyhydroxyalkanoates (PHAs) Synthesized by a New Strain, Cupriavidus necator IBP/SFU-1, from Various Carbon Sources. Polymers 2021, 13, 3142. https://doi.org/ $10.3390 /$ polym 13183142

Academic Editor: Esther Rebollar

Received: 8 August 2021

Accepted: 14 September 2021

Published: 17 September 2021

Publisher's Note: MDPI stays neutral with regard to jurisdictional claims in published maps and institutional affiliations.

Copyright: (c) 2021 by the authors. Licensee MDPI, Basel, Switzerland. This article is an open access article distributed under the terms and conditions of the Creative Commons Attribution (CC BY) license (https:// creativecommons.org/licenses/by/ $4.0 /)$
Abstract: The bacterial strain isolated from soil was identified as Cupriavidus necator IBP/SFU-1 and investigated as a PHA producer. The strain was found to be able to grow and synthesize PHAs under autotrophic conditions and showed a broad organotrophic potential towards different carbon sources: sugars, glycerol, fatty acids, and plant oils. The highest cell concentrations $(7-8 \mathrm{~g} / \mathrm{L})$ and PHA contents were produced from oleic acid (78\%), fructose, glucose, and palm oil (over $80 \%)$. The type of the carbon source influenced the PHA chemical composition and properties: when grown on oleic acid, the strain synthesized the $\mathrm{P}(3 \mathrm{HB}-\mathrm{co}-3 \mathrm{HV})$ copolymer; on plant oils, the $\mathrm{P}(3 \mathrm{HB}-\mathrm{co}-3 \mathrm{HV}-\mathrm{co}-$ $3 \mathrm{HHx}$ ) terpolymer, and on the other substrates, the $\mathrm{P}(3 \mathrm{HB})$ homopolymer. The type of the carbon source influenced molecular-weight properties of PHAs: $\mathrm{P}(3 \mathrm{HB})$ synthesized under autotrophic growth conditions, from $\mathrm{CO}_{2}$, had the highest number-average $(290 \pm 15 \mathrm{kDa})$ and weight-average $(850 \pm 25 \mathrm{kDa})$ molecular weights and the lowest polydispersity $(2.9 \pm 0.2)$; polymers synthesized from organic carbon sources showed increased polydispersity and reduced molecular weight. The carbon source was not found to affect the degree of crystallinity and thermal properties of the PHAs. The type of the carbon source determined not only PHA composition and molecular weight but also surface microstructure and porosity of the polymer films. The new strain can be recommended as a promising $\mathrm{P}(3 \mathrm{HB})$ producer from palm oil, oleic acid, and sugars (fructose and glucose) and as a producer of $\mathrm{P}(3 \mathrm{HB}-\mathrm{co}-3 \mathrm{HV})$ from oleic acid and $\mathrm{P}(3 \mathrm{HB}-\mathrm{co}-3 \mathrm{HV}-\mathrm{co}-3 \mathrm{HHx})$ from palm oil.

Keywords: Cupriavidus necator IBP/SFU-1; cell growth and PHA synthesis; various carbon sources; PHA composition and properties; polymer films

\section{Introduction}

Degradable polyhydroxyalkanoates (PHAs) are regarded as the real candidate to gradually replace non-degradable synthetic polymers, which are now used extensively, posing a global environmental problem [1-6]. Polymers of hydroxy-derived alkanoic acids, polyhydroxyalkanoates (PHAs), comprise polymers with various chemical compositions, which have valuable properties including biocompatibility and biodegradability. Therefore, these biopolymers are promising materials of the 21st century for diverse applications from municipal engineering and agriculture to pharmacology and biomedicine [7-12].

Polyhydroxyalkanoates are energy and carbon storage macromolecules of a cell, which are synthesized by prokaryotes under specific conditions of unbalanced growth when 
synthesis of the main compounds (protein and nucleic acids) is limited, but the medium contains excess carbon $[13,14]$. Conditions under which the direction of cell anabolism changes from protein synthesis to PHA synthesis and accumulation are determined by the redox state of cytoplasm and intracellular concentrations of pyruvate and available CoA [15]. During balanced growth of PHA-producing bacteria, pyruvate and reducing equivalents (NADH and NADPH) are mainly expended in the tricarboxylic acid cycle to form amino acids and to transform energy in the cell. The level of available CoA remains high, which hinders polymer synthesis. Under unbalanced growth, in a nitrogenfree or nutrient-deficient medium, pyruvate does not enter the tricarboxylic acid cycle but is carboxylated, forming acetyl-CoA. The level of available CoA is low, which is a favorable condition for activation of the enzymes involved in the intracellular cycle of storage macromolecules such as PHAs.

PHA production is a purely biological intracellular synthesis performed by bacterial cells grown on various carbon sources. PHAs are represented by polymers with different chemical structures and considerably diverse properties: molecular-weight and temperature characteristics, degree of crystallinity, and biodegradation behavior and rates [6,16-21]. PHAs may be potentially produced from substrates that can be reduced to different degrees and that vary in energy content and cost such as individual compounds and complex substrates, including industrial wastes [22]. Over 300 microorganisms are known to produce and accumulate PHAs, they are represented by both wild-type and genetically modified strains.

The hydrogen-oxidizing bacteria of the Cupriavidus genus (formerly Wautersia, Ralstonia, Alcaligenes, Hydrogenomonas) are promising PHA producers [23]. Being autotrophic, they are able to synthesize PHAs from mixtures of carbon dioxide and hydrogen [24-28]; moreover, they have a broad organotrophic potential and are capable of synthesizing PHAs with various chemical structures using diverse substrates [9,11,29-31]. Because of the strong intracellular system for PHA synthesis and special growth physiology, Cupriavidus strains synthesize very high PHA concentrations. Even on a complete nutrient medium, in the middle of the linear growth phase, these microorganisms stop synthesizing protein and begin producing poly-3-hydroxybutyrate (the first and best-studied PHA) [24,25]. However, the challenges of using most of the Cupriavidus species include their narrow trophic potential towards sugars and ability to assimilate only fructose for their growth. Another issue is that they are capable of synthesizing only short-chain-length PHAs-the $\mathrm{P}(3 \mathrm{HB})$ homopolymer and copolymers of $3 \mathrm{HB}$ and valerate, $\mathrm{P}(3 \mathrm{HB}-\mathrm{co}-3 \mathrm{HV})$. A usual way to overcome these limitations is to produce mutant organisms. Therefore, the isolation of highly productive wild-type strains, which have broader organotrophic potential and are able to synthesize PHA copolymers composed of short- and medium-chain-length monomers, can considerably enhance the potential of this genus. Despite the pressing need for degradable polymeric materials and the high attractiveness of PHAs, their high cost and the complications associated with technical and technological processes limit their manufacturing scale and reduce their applications [32,33]. The major challenge of PHA biotechnology is optimizing the entire biotechnology of PHA synthesis, primarily, by using new productive strains, which are capable of growing not only on sugars but also on various available carbon sources, including inexpensive plant oils, glycerol (which is a by-product of large-scale biodiesel production), etc., and are able to synthesize PHA copolymers with different chemical compositions, containing short- and medium-chain-length monomers and showing better performance.

The present study investigated the properties of PHAs synthesized by the new strain, Cupriavidus necator IBP/SFU-1, from various carbon sources. 


\section{Materials and Methods}

\subsection{Isolation of the Strain}

The strain is maintained in the Collection of Chemolithotrophic Cultures of the Laboratory of Chemoautotrophic Biosynthesis at the Institute of Biophysics SB RAS. The strain was isolated from soil (Krasnoyarsk, Siberia, Russia).

\subsection{Identification of the Bacterial Strain}

The bacterial strain was identified using conventional methods based on the culture and morphological characters, standard biochemical tests [34,35], sequencing of a region of the 16S rRNA gene, and the Matrix-Assisted Laser Desorption/Ionization Time-of-Flight (MALDI-TOF) mass spectrometry method, performed using MALDI-TOF MS Microflex (Bruker Daltonics, Bremen, Germany), based on the automatic analysis of mass spectra of protein molecules specific for the microorganism species.

Phylogenetic analysis of the strain was performed using the Neighbor-Joining method [36]. The evolutionary distances were computed using the Maximum Composite Likelihood method [37] and were in the units of the number of base substitutions per site. Codon positions included were $1 \mathrm{st}+2 \mathrm{nd}+3 \mathrm{rd}+$ Noncoding. All ambiguous positions were removed for each sequence pair (pairwise deletion option). Phylogenetic and molecular evolutionary analyses were conducted using the MEGA software package version X [38].

\subsection{Cultivation of Cupriavidus Necator IBP/SFU-1 Cells}

The aseptic cultivation of bacterial cells was conducted under autotrophic growth conditions in Schlegel's mineral medium, on the $\mathrm{CO}_{2}: \mathrm{O}_{2}: \mathrm{H}_{2}$ gas mixture $(1: 2: 7 v / v \%)$. Under heterotrophic growth conditions, the medium contained one of the following carbon and energy sources at a concentration of 10-15 g/L: fructose (Panreac, Barcelona, Spain) and glucose (Khimreactivsnab, Ufa, Russia); purified glycerol (Corporate Oleon, Emmerich, Germany); plant oils including refined sunflower seed oil and Siberian oilseed oil ("Zolotaya semechka", Rostov-on-Don, Russia) and bleached refined deodorized palm oil ("Oil de Luxe", Surabaya, Indonesia); fatty acids including palmitic (Vekton, Russia), myristic (Merck, Bandar Sunway, Malaysia), lauric (Merck, Malaysia), and oleic (Ekos-1, Moscow, Russia) acids.

Fermentation was conducted in 0.5-L flasks containing $200 \mathrm{~mL}$ of the medium in an Innova 44 constant temperature incubator shaker ("New Brunswick Scientific", Edison, NJ, USA) at $200 \mathrm{rpm}$ and $30{ }^{\circ} \mathrm{C}$. The cell cultivation process was described in detail elsewhere [39]; production parameters of the culture such as cell concentration $(\mathrm{X}, \mathrm{g} / \mathrm{L})$, intracellular polymer content $(\mathrm{g} / \mathrm{L}$ and $\%$ of $\mathrm{CDW})$, and yield coefficients from the carbon source $(\mathrm{Y}, \mathrm{g} / \mathrm{g})$ were determined using conventional methods.

Biomass fermentation productivity was determined using the formula:

$$
\mathrm{P}_{\mathrm{X}}=\left(\mathrm{X}_{\mathrm{n}}-\mathrm{X}_{0}\right) / \mathrm{T},
$$

where $X_{0}, X_{n}$ are concentrations of biomass at the start and end of fermentation, respectively, $\mathrm{g} / \mathrm{L}, \mathrm{T}$ is fermentation duration $\mathrm{h}^{-1}$.

The yield coefficients of the biomass and polymer, $Y_{x}$ and $Y_{p}$, from the substrates were calculated using the following formula:

$$
\mathrm{Y}_{\mathrm{X}}=\Delta \mathrm{X} / \Delta \mathrm{S}, \mathrm{Y}_{\mathrm{P}}=\Delta \mathrm{PHA} / \Delta \mathrm{S},
$$

where $\Delta \mathrm{X}$ and $\Delta \mathrm{P}$ are the difference between the initial and final biomass and polymer content, $\mathrm{g}$, and $\Delta \mathrm{S}$ is the consumed substrate, $\mathrm{g}$.

\subsection{Analytical Procedures}

The intracellular content and composition of PHAs were determined by chromatography of the preliminarily derived methyl ester fatty acids using gas chromatography-mass spectrometry (GC-MS) (7890/5975C, Agilent Technologies, Santa Clara, CA, USA) [40]. 
The lipids were extracted from wet biomass with the chloroform-methanol mixture $(2: 1, v / v)$. Cells grown on the medium with plant oils were subjected to preliminary treatment with hexane to remove the substrates. In the resulting extract, PHAs were separated from the lipids by precipitation with a double volume of hexane. The lipid extract was dried, and after methyl esterification with $\mathrm{H}_{2} \mathrm{SO}_{4}$ :methanol (1:20) solution that lasted $2 \mathrm{~h}$ at $80^{\circ} \mathrm{C}$, fatty acid methyl esters (FAMEs) were analyzed using GC-MS (7890/5975C, Agilent Technologies, USA).

\subsection{PHA Properties}

The physicochemical properties of high-purity PHA specimens were determined using modern physicochemical methods, which have been described in detail elsewhere [41,42]. Molecular-weight properties of the polymers (weight-average $\left(\mathrm{M}_{\mathrm{w}}\right)$ and number-average $\left(\mathrm{M}_{\mathrm{n}}\right)$ molecular weights and polydispersity $\left.(Đ)\right)$ were measured. Melting point $\left(\mathrm{T}_{\text {melt }}\right)$ and thermal degradation temperature (temperature at which sample mass loss begins, $\mathrm{T}_{\text {degr }}$ ) were measured using a DSC-1 differential scanning calorimeter (Mettler Toledo, Schwerzenbac, Switzerland) and TGA (Mettler Toledo, Schwerzenbac, Switzerland), respectively. A 3-5 mg sample was heated to $200{ }^{\circ} \mathrm{C}$ at a rate of $5{ }^{\circ} \mathrm{C} / \mathrm{min}$; the sample was held at $200{ }^{\circ} \mathrm{C}$ for $1 \mathrm{~min}$, cooled to $-20^{\circ} \mathrm{C}$ at a rate of $5^{\circ} \mathrm{C} / \mathrm{min}$ and held for $4 \mathrm{~min}$. Then, the sample was reheated at a rate of $5{ }^{\circ} \mathrm{C} / \mathrm{min}$ (DSC). A 3-5 mg sample was heated to $450{ }^{\circ} \mathrm{C}$ at a rate of $10^{\circ} \mathrm{C} / \mathrm{min}$ (TGA). Thermograms were analyzed using the "StarE"software. X-ray examination was performed using a D8ADVANCE powder diffractometer (Bruker AXS, Karlsruhe, Germany) equipped with a VANTEC linear detector; the degree of crystallinity $\left(C_{x}\right)$ was calculated as a ratio of the total area of crystalline peaks to the total area of the radiogram (the crystalline + amorphous components

\subsection{Preparation and Investigation of Polymer Films}

Films were prepared by casting a $2 \%$ polymer solution in dichloromethane in degreased Teflon-coated molds, and then the films were left to stay in a laminar flow cabinet (Labconco, Kansas City, MO, USA) for $72 \mathrm{~h}$ at room temperature. The surface microstructure of PHA films was analyzed using scanning electron microscopy (FE-SEM S 5500 high-resolution scanning electron microscope Hitachi, Tokyo, Japan). Prior to microscopy, the samples were sputter-coated with platinum (at $25 \mathrm{~mA}$, for $60 \mathrm{~s}$ ), using an EM ACE200 ("Leica", Wetzlar, Germany). Water contact angles of the films were studied with a Drop Shape Analyzer-DSA-25E (Krüss, Hamburg, Germany) using the DSA-4 software for Windows. Porosity of the films was determined manually from SEM images using a software package for digital image analysis (free open-source software package for scientific analysis, editing, and processing of raster images), Image J v1.53d.

\subsection{Statistics}

Statistical analysis of the results was performed using the standard software package of Microsoft Excel. Arithmetic means and standard deviations were found.

\section{Results and Discussion}

\subsection{Characterization of the Strain Cupriavidus Necator IBP/SFU-1}

The following morpho-cultural characteristics of Cupriavidus necator IBP/SFU-1 were determined. The cells were motile $0.3-0.5 \times 1.2-2.0-\mu \mathrm{m}$ rods, which formed cream-colored, smooth colonies on peptone agar. The colonies had the obvious single-center morphology and the colony edge was smooth. The diameter of the 7-day colonies was 3-5 mm, with their number per Petri dish no more than 10.

Biochemically, Cupriavidus necator IBP/SFU-1 is an obligate aerobic organism and a facultative chemolitho-organotroph; the strain is capable of growing on $\mathrm{CO}_{2}$ and $\mathrm{H}_{2}$ as sole carbon and energy sources, respectively. As a nitrogen source, the strain utilizes nitrates, ammonium salts, urea, and amino acids. It is oxidase positive. The strain does not have hydrolytic enzymes, does not liquefy gelatin, and does not hydrolyze starch. Of the tested 
sugars, the strain utilizes fructose and glucose. It is capable of growing on the media with organic acids and amino acids. It does not need growth factors; it is an antibiotic-resistant strain. The optimal growth temperature is $30-33{ }^{\circ} \mathrm{C}, \mathrm{pH} 7.0 \pm 0.2$.

Identification of the composition of lipid fatty acids, which is a taxonomic characteristic, shows that the FAs of the strain are represented by the fatty acids containing 12 to 19 carbons, palmitic acid being the predominantly saturated acid (46.2\%). Palmitoleic (16:1 $\omega 7)$ and cis-vaccenic acids are the main monoenoic acids, constituting 18.3-27.6 and $22.9-24.2 \%$, respectively. The saturated/unsaturated FA ratio is 0.9 under autotrophic conditions and 1.4 on fructose (data not shown).

In Cupriavidus necator IBP/SFU-1, the nucleotide sequence of the $16 \mathrm{~S}$ rNA gene contained $1482 \mathrm{bp}$. The obtained nucleotide sequence of the 16S rNA gene was deposited in the GenBank database (Accession Number MW680848.1).

The phylogenetic position of strain IBP/SFU-1 within the genus Cupriavidus is shown in Figure 1. Phylogenetic analysis showed that the strain IBP/SFU-1 represented a member of the genus Cupriavidus. Comparative 16S rRNA gene sequence analysis revealed that the strain IBP/SFU-1 had a high degree of similarity with other species of the genus Cupriavidus.

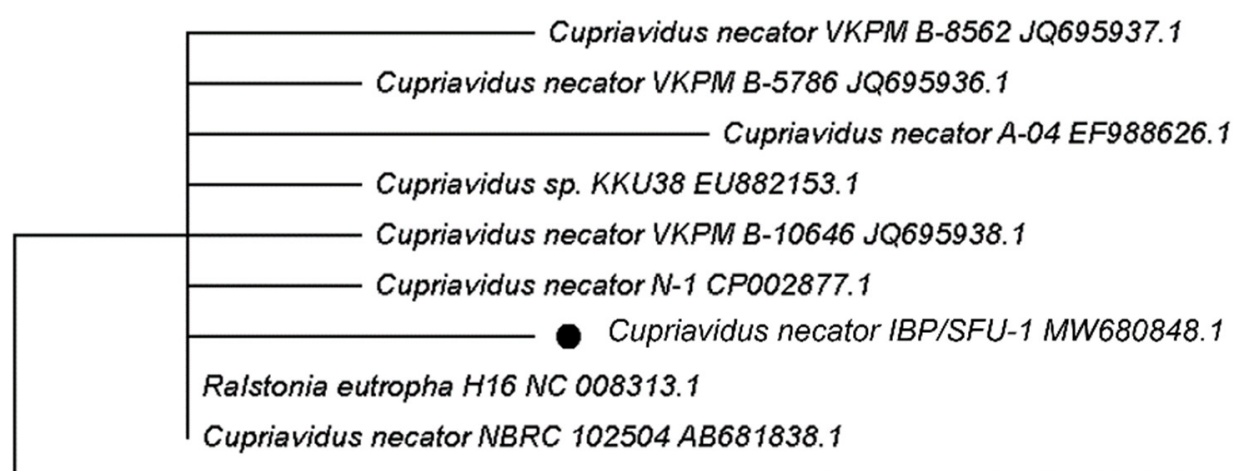

Cupriavidus sp. MA112 MH699182.1

Figure 1. Neighbor-joining phylogenetic tree of Cupriadivus necator IBP/SFU-1 and related bacteria based on 16S rRNA sequence comparisons. Accession numbers are given. Bar, 0.0005 substitutions per nucleotide position.

\subsection{Cupriavidus Necator IBP/SFU-1 Growth and PHA Synthesis on Various Carbon Sources}

The study showed that the strain C. necator IBP/SFU-1 was capable of growing and synthesizing PHAs on various carbon sources: $\mathrm{CO}_{2}$, sugars, glycerol, fatty acids, and plant oils. Hence, there are different PHA synthesis pathways in this strain. Eight possible biochemical pathways of PHA synthesis were described in the review by Professor Chen [16]. The first pathway, involving three key enzymes, $\beta$-ketothiolase, NADPHdependent acetoacetyl-CoA reductase, and PHA synthase, occurs in hydrogen-oxidizing bacteria including Cupriavidus necator. This pathway was also found in Aeromonas hydrophila, Pseudomonas stutzeri, and Pseudomonas oleovorans [16].

Production parameters of the batch culture of C. necator IBP/SFU-1 grown on different carbon sources are shown in Figure 2. The strain was found to be able to synthesize PHAs in autotrophic suspension culture with $\mathrm{CO}_{2}$ as the sole carbon source and $\mathrm{H}_{2}$ oxidation reaction as the energy source. Cell biomass concentration and polymer content in the three-day culture were $2.6 \mathrm{~g} / \mathrm{L}$ and $35.1 \%$, respectively. That was comparable to the corresponding parameters achieved using Cupriavidus necator Z-1 [25] but inferior to the parameters of the strains maintained in the collection of the Institute of Biophysics SB RAS, Cupriavidus necator B5786 and Cupriavidus necator B-10646, which, when grown under the same conditions, produced $3.5-4.0 \mathrm{~g} / \mathrm{L}$ of cell biomass and $60-70 \%$ (or even higher) polymer content $[28,43]$. However, C. necator IBP/SFU-1 is a newly isolated strain, which has been mainly maintained in the stock culture on agar medium. In contrast to Cupriavidus necator B 5786 and C. eutrophus B-10646, it has not been used in suspension culture for 
long periods, has not been selected based on its growth rate, and has not been adapted to the mode of PHA accumulation on $\mathrm{CO}_{2}$. Therefore, further research may lead to the enhancement of production parameters of $\mathrm{C}$. necator IBP/SFU-1.
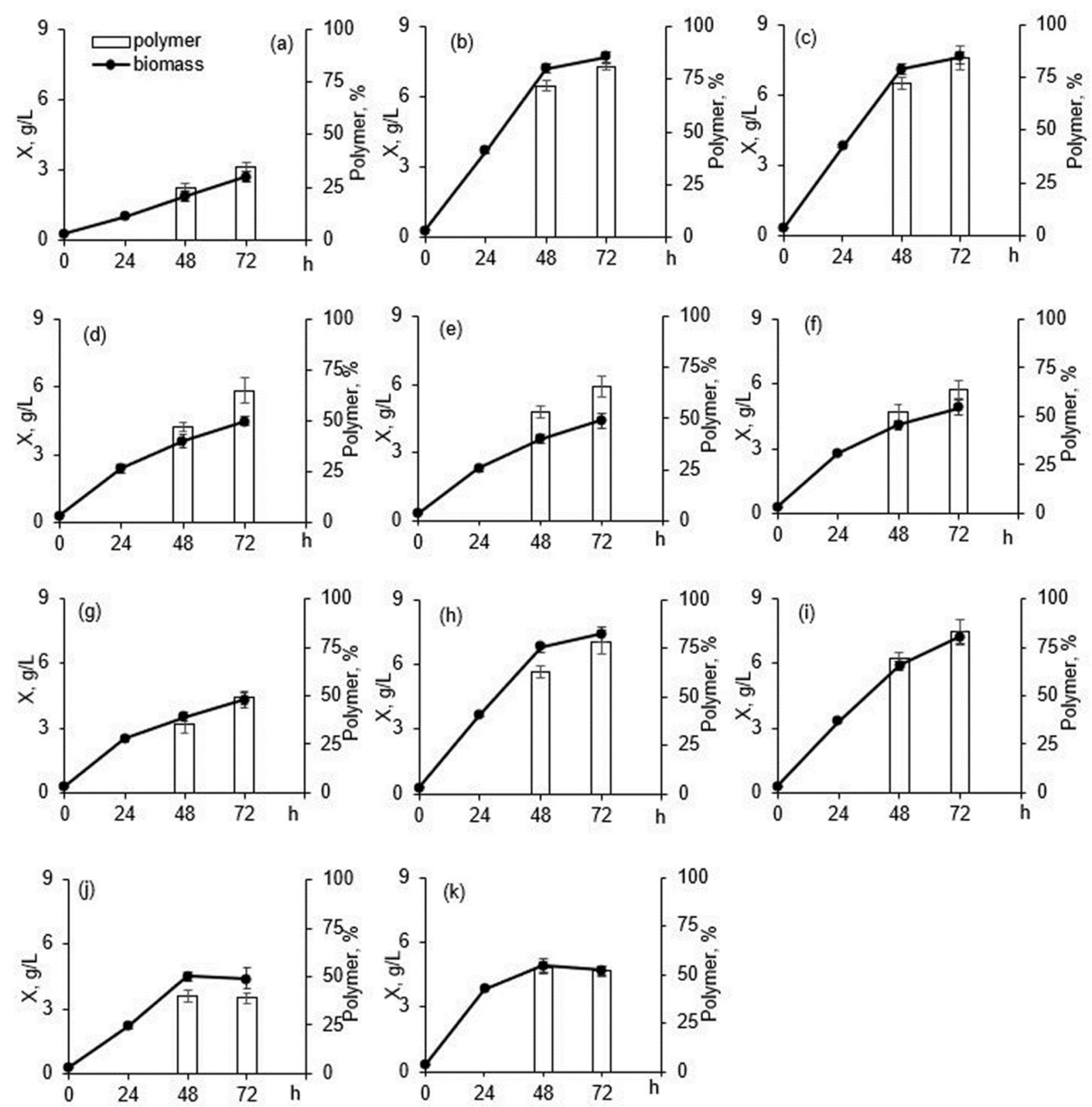

Figure 2. Cell dry weight and PHA production by Cupriavidus necator IBP/SFU-1 grown on different substrates. (a) $\mathrm{CO}_{2}$, (b) fructose, (c) glucose, (d) glycerol, (e) lauric acid, (f) myristic acid, (g) palmitic acid, (h) oleic acid, (i) palm oil, (j) sunflower seed oil, (k) Siberian oilseed oil.

The new strain, C. necator IBP/SFU-1, has a broad organotrophic potential and is able to synthesize high PHA concentrations from various organic substrates such as sugars, glycerol, fatty acids (FAs), and plant oils (Figure 2).

Sugars are the most common substrate for a variety of microorganisms, including PHA producers, which are able to metabolize hexoses for growth. Although the only sugar utilized by hydrogen-oxidizing bacteria is fructose, they readily undergo mutations and acquire the ability to metabolize glucose. The carbohydrate that is generally used to reach high PHA concentrations is ketohexose (fructose), which is phosphorylated by fructokinase into fructose-6-phosphate, which is then isomerized to glucose-6-phosphate, transformed 
to phosphogluconate, and metabolized via the Entner-Doudoroff pathway. When bacterial cells are grown on glucose instead of fructose, glucose is metabolized via the EntnerDoudoroff pathway to form pyruvate, which may be transformed by dehydrogenase into acetyl-CoA, the central intermediate component in reactions of PHA synthesis [44].

The strain C. necator IBP/SFU-1 was found to be capable of metabolizing glucose, and there was no need to go through a lengthy process of isolation on the selective medium with glucose as the sole carbon source, in contrast to the production of the glucose-utilizing mutant strain Cupriavidus necator B 8562 from the parent strain Cupriavidus necator B 5786 [45]. Cultivation of C. necator IBP/SFU-1 on fructose and glucose resulted in similar cell concentrations and PHA contents: cell concentrations were approximately 7.0 and $7.7 \mathrm{~g} / \mathrm{L}$ (productivity of 2.33 and $2.57 \mathrm{~g} / \mathrm{L} \mathrm{d}$, respectively), and PHA contents were $81.1(5.68 \mathrm{~g} / \mathrm{L})$ and $84.3 \%(6.33 \mathrm{~g} / \mathrm{L})$. These values were slightly lower than the results achieved in the 72-h cultivation of the highly productive strain C. necator B-10646, whose cell concentration reached $8.4 \mathrm{~g} / \mathrm{L}$ and PHA content $92 \%$ [43], and similar to, or higher, than the results reported by other authors for such strains as Cupriavidus necator H16 [46,47], Cupriavidus necator NCIMB 11599 [48], Cupriavidus necator B 8562 [45], and Cupriavidus necator A-04 [30]. The new strain consumed similar amounts of fructose and glucose, $2.8 \pm 0.2 \mathrm{~g} / \mathrm{g}$ cell biomass and $3.3 \pm 0.3 \mathrm{~g} / \mathrm{g}$ polymer, and yield coefficients were $\mathrm{Y}_{\mathrm{x}} 0.36 \pm 0.03$ and $\mathrm{Y}_{\mathrm{PHA}} 0.30 \pm 0.03$.

A promising source for PHA production by various bacteria, including Cupriavidus, is glycerol, a byproduct of the large-scale and steadily growing biodiesel production [49]. In contrast to glucose, which is metabolized via the Entner-Doudoroff pathway to form pyruvate, which is then transformed by dehydrogenase into acetyl-CoA, glycerol can also be metabolized to form pyruvate but through the intermediate compound, glyceraldehyde3-phosphate [50].

The ability of the strain C. necator IBP/SFU-1 to synthesize PHA from glycerol was investigated in experiments with purified glycerol (containing $0.3 \%$ impurities) because PHA accumulation on glycerol is largely determined by the degree of the purity of glycerol and the number of impurities such as methanol, salts, and esters of fatty acids, etc., which can considerably inhibit cell growth. Cultivation of C. necator IBP/SFU-1 on purified glycerol resulted in a cell concentration of $5.0 \mathrm{~g} / \mathrm{L}$ (productivity of $1.67 \mathrm{~g} / \mathrm{L} \cdot \mathrm{d}$ ) and polymer content of $65.1 \%(3.26 \mathrm{~g} / \mathrm{L})$ (Figure 2$)$. Cell biomass and polymer yield coefficients were $\mathrm{Y}_{\mathrm{X}}=0.38 \pm 0.02$ and $\mathrm{Y}_{\mathrm{PHA}}=0.29 \pm 0.01 \mathrm{~g} / \mathrm{g}$, respectively.

C. necator IBP/SFU-1 cells grown on glycerol in the medium inoculated with the stock culture maintained on the nutrient agar did not show any lag phase. By contrast, three wild-type strains, C. necator B 5786, B 8562, and B-10646, showed 20- to 30-40-h lag phases regardless of the initial glycerol concentration in the medium. The 80-h cultivation resulted in a $C$. necator $\mathrm{B}-10646$ cell concentration of $2.2 \mathrm{~g} / \mathrm{L}$, and that was higher than cell concentrations of C. necator B 5786 (0.7-0.8 g/L) and C. necator B 8562 (1.0-1.1 g/L); polymer concentrations also differed across the strains: 57,45 , and $38 \%$, respectively. Only after the strains were adapted to glycerol by numerous re-inoculations, did the cells not show any lag phase in the early growth phases, and cell concentrations and polymer contents increased to 5.1 and $6.2 \mathrm{~g} / \mathrm{L}$ and 57 and $71 \%$, respectively [39]. Thus, production parameters of $C$. necator IBP/SFU-1 grown on glycerol were comparable to the results of cultivation of the strain C. necator B-10646, which was adapted to glycerol, and higher than the parameters obtained under similar conditions for other wild-type strains: $C$. necator B 5786 and B 8562, C. necator IPT 026, Ps. oleovorans NRRLB-14682, Ps. corrugate 388, and Paracoccus sp. LL1 [51-53].

PHA synthesis by C. necator IBP/SFU-1 was also investigated in experiments with fatty acids (FAs) used as the growth substrate. Metabolism of fatty acids was described as a second pathway of PHA synthesis by microorganisms in a review by Chen [16]. This pathway suggests that after $\beta$-oxidation of fatty acids, acyl-CoA is involved in the synthesis of monomers, which are then catalyzed by PHA-synthase in polymerization reactions to produce polymers. The synthesis of 3-hydroxyacyl-CoA involves 3-ketoacylCoA-reductases, epimerase, and, presumably, (R)-enoyl-CoA-hydratase, acyl-CoA-oxidase, 
and enoyl-CoA-hydratase I. This pathway is typical for the synthesis of medium-chainlength PHAs such as copolymers of 3-hydroxybutyrate and 3-hydroxyhexanoate.

Not only fatty acids but also spent cooking fats and products based on them and various plant oils, are potentially promising carbon sources for PHA synthesis. These substrates are rich in carbon and are efficiently converted to PHAs [54-59].

C. necator IBP/SFU-1 cell growth and PHA synthesis were studied in cultures with unsaturated (lauric, myristic, and palmitic) acids and an unsaturated, oleic, acid (Figure 2). Cultivation on oleic acid resulted in the highest cell concentration and intracellular PHA content: $8.0 \mathrm{~g} / \mathrm{L}$ with a productivity of $2.67 \mathrm{~g} / \mathrm{L} \mathrm{d}$ and $78.4 \%(6.27 \mathrm{~g} / \mathrm{L})$. Those values were comparable to the corresponding parameters reported for C. necator B-10646 [60] and other strains including Alcaligenes sp. NCIM N5085 [61,62] grown on the same FA. Polymer concentration synthesized by $C$. necator IBP/SFU-1 was higher but cell concentration was lower than those reported for C. necator USMAHM13 [63].

When grown on any of the saturated FAs, C. necator IBP/SFU-1 produced cell concentrations comparable to those achieved on oleic acid, but polymer concentrations were lower (Figure 2): 65.9 and 64.2\% on lauric and myristic acids, respectively, and only $49 \%$ on palmitic acid. Similarly lower parameters were obtained on saturated FAs compared to oleic acid in studies of $C$. necator USMAHM13 [63] and C. necator USMAA2-4 [62]. The most likely explanation lies in the physiological and biochemical properties of each strain and its ability to metabolize a certain fatty acid. For the strain C. necator USMAA2-4, FAs, as effective substrates for bacterial cells, were ranked as follows: oleic acid > palmitic acid $>$ myristic acid > lauric acid [62]. For C. necator USMAHM13, by contrast, cells grown on palmitic acid showed the lowest biomass concentration and PHA content [63].

Three plant oils, palm oil, Siberian oilseed oil, and sunflower seed oil, were tested as carbon sources for C. necator IBP/SFU-1; the oils differed considerably in their FA compositions as shown in a study by Volova et al. (2020) [64]. Sunflower seed oil was

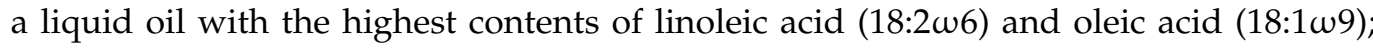
other acids (20:0, 22:0, and 24:0) were present in small amounts (0.4-1.1\%); the saturation index of the oil was 0.14 . Siberian oilseed oil contained 15 FAs, and unsaturated FAs were prevalent as well (constituting about 90\%); its saturation index was similar to that of refined sunflower seed oil (0.11). Palm oil was a semisolid or solid plant oil; the saturation index of the tested sample was 0.97 , suggesting the prevalence of saturated FAs. Thus, the tested oils differed considerably in their FA compositions.

Cultivation of $C$. necator IBP/SFU-1 on palm oil resulted in the highest production parameters (Figure 2). Cell concentration was $8.2 \mathrm{~g} / \mathrm{L}$ (with the highest productivity of $2.73 \mathrm{~g} / \mathrm{L} \cdot \mathrm{d}$ ) and intracellular polymer content was $80 \%(6.56 \mathrm{~g} / \mathrm{L})$. That was comparable with the results obtained in the 72-h culture of the wild-type strain C. necator B-10646-7.1 g/L and 7-82.7\%, respectively [64], and superior to the production parameters of another wild-type strain, C. necator H16-5.6 g/L and 77\%, respectively [65]. Not all C. necator strains, however, are able to utilize palm oil, the wild-type strain C. necator USMAA2-4 failed to grow on palm oil [62].

Results of C. necator IBP/SFU-1 cultivation on sunflower seed oil were lower compared to palm oil and were similar to the results obtained for C. necator B-10646: a cell concentration of $4.3 \mathrm{~g} / \mathrm{L}$, a productivity of only $1.77 \mathrm{~g} / \mathrm{L} \mathrm{d}$, and a polymer content of $38.7 \%$ [64]. Lee et al. (2008) reported a similar cell concentration but higher polymer content (72\%) for C. necator H16 [65]. In another study [66], however, cell concentration produced by the same strain, H-16, was higher $(9.4 \mathrm{~g} / \mathrm{L})$ but polymer content lower $(49 \%)$.

Siberian oilseed oil was first tested as a source for PHA synthesis in a culture of C.necator B-10646 [64]; the resulting cell concentration was $5.8 \mathrm{~g} / \mathrm{L}$ and polymer content $59 \%$. In the present study, C. necator IBP/SFU-1 produced similar results. All fat-containing sources were suitable substrates for the growth of the new strain and for PHA synthesis, although production parameters of the cultures differed somewhat. The yield coefficients for palm oil, Siberian oilseed oil, and fatty acids were $0.76 \pm 0.01 \mathrm{~g}$ biomass $/ \mathrm{g}$ substrate and $0.48 \pm 0.02 \mathrm{~g}$ polymer/g substrate, and those values were 1.5-2.0 times higher than the yield coefficients obtained for sunflower seed oil, sugars, and glycerol. 
Our results showed that the newly isolated wild-type strain Cupriavidus necator IBP/SFU-1 is able to grow and synthesize PHAs not only on the highly specialized fructose but also on the more available glucose, fatty acids, various plant oils, and glycerol as well as under autotrophic growth conditions, on $\mathrm{CO}_{2}$ mixed with hydrogen and oxygen. Moreover, the replacement of the sugar sources by any of the other carbon sources tested in the present study did not lead to any long adaptation or lag-phase. This is a definite advantage of the new strain over the other known Cupriavidus representatives, some of which are unable to assimilate glucose or palm oil, show low productivity on glycerol, and need a long time to adapt to other carbon sources after being reinoculated from sugar-containing media.

\subsection{Properties of PHAs Synthesized by Cupriavidus Necator IBP/SFU-1 from Various Carbon Sources}

Properties of PHAs are determined by their structure, primarily, the structure of the side chains in the polymer carbon chain and the distance between the ester groups in a molecule $[10,11,14,16]$. The ability of the PHA producing strains to synthesize polymers with different compositions is determined by their biochemical properties, organization of the intracellular enzyme system of PHA synthesis, and substrate specificity of PHAsynthase-the enzyme catalyzing monomer polymerization reactions. The factor of the culture medium that influences the chemical composition of the PHAs is the carbon source.

It was previously believed that Cupriavidus species were only able to synthesize shortchain length PHAs: $\mathrm{P}(3 \mathrm{HB})$ and $\mathrm{P}(3 \mathrm{HB}-\mathrm{co}-3 \mathrm{HV})$. Cupriavidus strains had been considered as producers of short-chain-length PHAs (scl-PHAs) until some of the strains of this genus were found to be able to synthesize PHAs containing medium-chain-length monomers (3-hydroxyhexanoate, 3-hydroxyoctanoate) when cultivated in the medium supplemented with the corresponding precursor substrates $[28,29]$. The ability to synthesize a wide range of PHAs including short- and medium-chain-length monomers makes these strains particularly promising PHA producers.

Cultivation of $C$. necator IBP/SFU-1 on various carbon sources resulted in differences in not only production parameters and substrate utilization efficiency but also the composition and properties of the synthesized polymers. Ion chromatograms and mass spectra of the polymers synthesized by C. necator IBP/SFU-1 from various carbon sources provide evidence for the effect of the type of the carbon source on PHA composition (Figure 3). Cultivation of the strain on oleic acid and all plant oils resulted in the synthesis of PHA copolymers. The $\mathrm{P}(3 \mathrm{HB}-\mathrm{co}-3 \mathrm{HV})$ copolymers containing 3-hydroxyvalerate $(3 \mathrm{HV})$ monomers as the minor component of the carbon chain of the dominant 3-hydroxybutyrate $(3 \mathrm{HB})$ were synthesized from oleic acid. The $\mathrm{P}(3 \mathrm{HB}-\mathrm{co}-3 \mathrm{HV}-\mathrm{co}-3 \mathrm{HHx})$ terpolymers composed of $3 \mathrm{HB}$ (above 99\%) and minor fractions of 3-hydroxyvalerate (3HV) and medium-chain-length 3-hydroxyhexanoate (3HHx) were synthesized from three plant oils (Table 1). Those results were consistent with the data reported in another study [64]. In that study, the wild-type strain C. necator B-10646, which when grown on sugars synthesized the $\mathrm{P}(3 \mathrm{HB})$ homopolymer, was grown on plant oils (palm oil, Siberian oilseed oil, and sunflower seed oil) and produced a PHA terpolymer composed of the dominant 3HB (97-99 mol.\%) and the minor fractions of 3-hydroxyvalerate monomers (0.9-1.9 mol.\%) and the medium-chain-length 3-hydroxyhexanoate (0.4-1.3 mol.\%).

The main differences in the physicochemical properties of the polymers were between their molecular-weight properties-a major characteristic of high-molecular-weight compounds, affecting polymer processing into products and their mechanical qualities (Figure 4, Table 1). $\mathrm{P}(3 \mathrm{HB})$ synthesized under autotrophic growth conditions on $\mathrm{CO}_{2}$ had the highest molecular weight $\left(\mathrm{M}_{\mathrm{n}}\right.$ and $\left.\mathrm{M}_{\mathrm{w}}\right)$ and decreased polydispersity $(Đ)$. The other $\mathrm{P}(3 \mathrm{HB})$ specimens, which were synthesized from organic sources, showed reduced molecular weight and increased polydispersity. These results are consistent with the data on other wild-type strains of hydrogen-oxidizing bacteria (C. necator B 5786, C. necator B 8562, C. necator B-10646), although $\mathrm{P}(3 \mathrm{HB})$ synthesized on $\mathrm{CO}_{2}$ by those strains had much higher absolute values of weight-average molecular weight, reaching $1000 \mathrm{kDa}$ and even more [46]. The present study showed a decrease of 200 and even $350 \mathrm{kDa}$ in the 
$\mathrm{M}_{\mathrm{w}}$ of $\mathrm{P}(3 \mathrm{HB})$ synthesized by $C$. necator IBP/SFU-1 from fatty acids and plant oils and an increase in $Đ$ to 3.2-4.5 compared to the results obtained on $\mathrm{CO}_{2}$. A previous study demonstrated [64], that although polydispersity of the PHAs synthesized by C. necator B-10646 grown on plant oils increased considerably (to 4-5), their $\mathrm{M}_{\mathrm{w}}$ values ranged between 670 and $780 \mathrm{kDa}$, and the PHAs synthesized from palm oil had the lowest molecular weight. It is likely that the decrease in molecular weight of PHAs synthesized by bacterial cells grown on oils was caused by the metabolism of triacylglycerols in the culture medium containing oil and the release of glycerol, which functioned as a chain transfer agent during PHA polymerization [67], resulting in a lower-molecular-weight polymer. The most considerable reduction in the molecular weight of the $\mathrm{P}(3 \mathrm{HB})$ specimens synthesized by C. necator IBP/SFU-1 from glycerol supports this suggestion.
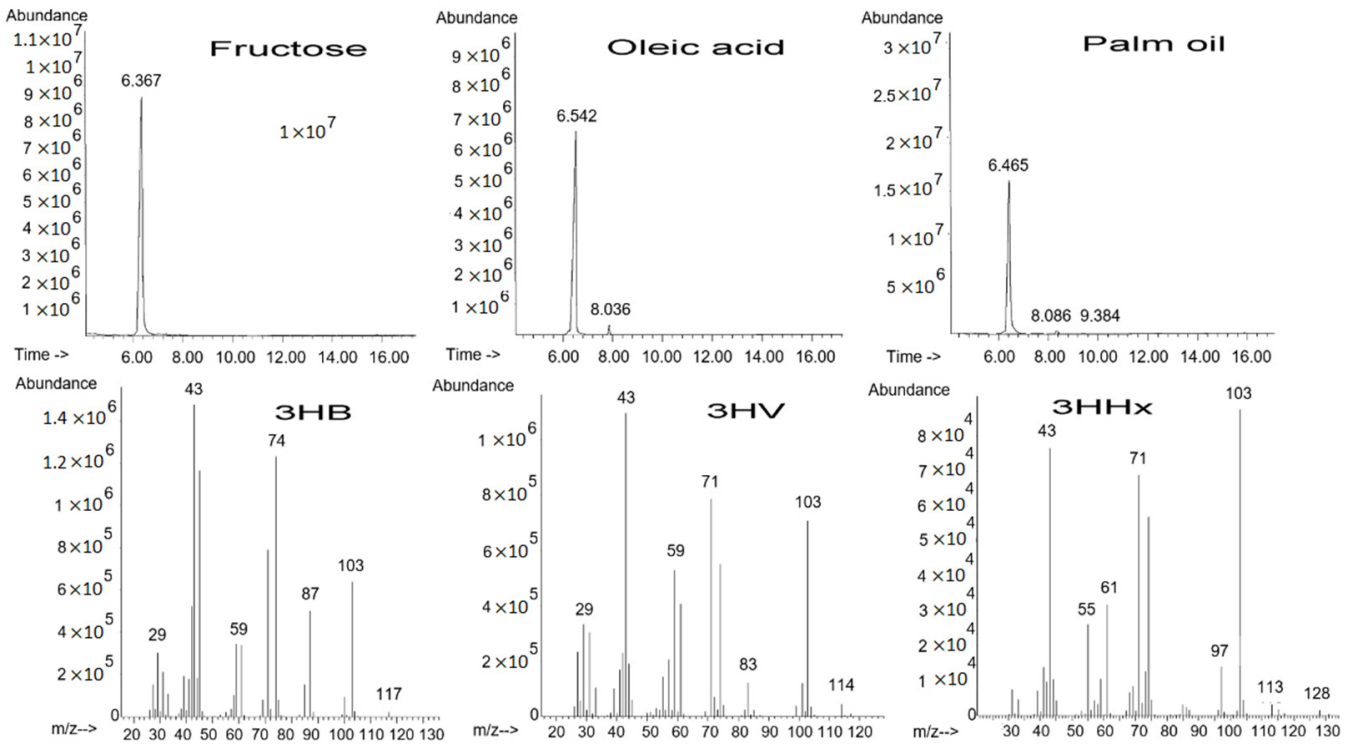

Figure 3. Ion chromatograms with mass spectra of methyl esters of the monomers constituting PHAs synthesized by Cupriavidus necator IBP/SFU-1 from different carbon sources with retention times: methyl-3-hydroxybutyrate (3HB) -6.367-6.542; methyl-3-hydroxyvalerate (3HV)—8.036-8.086; methyl-3-hydroxyhexanoate—9.384 min. $1.1 \times 10$.

Table 1. Composition and properties of PHAs synthesized by Cupriavidus necator IBP/SFU-1 from various carbon sources.

\begin{tabular}{|c|c|c|c|c|c|c|c|c|c|c|}
\hline \multirow[t]{2}{*}{ No. } & \multirow[t]{2}{*}{ Carbon Source } & \multicolumn{3}{|c|}{$\begin{array}{c}\text { Monomer Composition of } \\
\text { PHA, mol. } \%\end{array}$} & \multirow{2}{*}{$\mathrm{M}_{\mathrm{n}}, \mathrm{kDa}$} & \multirow{2}{*}{$\mathrm{M}_{\mathrm{w}}, \mathrm{kDa}$} & \multirow[t]{2}{*}{ Đ } & \multirow{2}{*}{$\mathrm{C}_{\mathrm{x},} \%$} & \multirow{2}{*}{$\mathrm{T}_{\text {melt }},{ }^{\circ} \mathrm{C}$} & \multirow[t]{2}{*}{$\mathrm{T}_{\text {degr } r}{ }^{\circ} \mathrm{C}$} \\
\hline & & $3 \mathrm{HB}$ & $3 \mathrm{HV}$ & $3 H H x$ & & & & & & \\
\hline 1 & $\mathrm{CO}_{2}$ & 100.0 & - & - & $290 \pm 15$ & $850 \pm 25$ & $2.9 \pm 0.2$ & 67 & 174.2 & 280.1 \\
\hline 2 & Fructose & 100.0 & - & - & $136 \pm 9$ & $499 \pm 20$ & $3.7 \pm 0.4$ & 65 & 172.4 & 284.7 \\
\hline 3 & Glucose & 100.0 & - & - & $137 \pm 1$ & $557 \pm 24$ & $4.1 \pm 0.2$ & 64 & 171.6 & 279.9 \\
\hline 4 & Glycerol & 100.0 & - & - & $108 \pm 4$ & $409 \pm 11$ & $3.9 \pm 0.3$ & 69 & 172.8 & 274.9 \\
\hline 5 & Lauric acid & 100.0 & - & - & $162 \pm 11$ & $510 \pm 10$ & $3.2 \pm 0.2$ & 63 & $\begin{array}{l}161.0 \\
170.6\end{array}$ & 279.0 \\
\hline 6 & Myristic acid & 100.0 & - & - & $144 \pm 10$ & $648 \pm 12$ & $4.5 \pm 0.3$ & 65 & $\begin{array}{l}162.1 \\
170.8\end{array}$ & 275.8 \\
\hline 7 & Palmitic acid & 100.0 & - & - & $174 \pm 8$ & $673 \pm 4$ & $3.9 \pm 0.2$ & 72 & $\begin{array}{l}162.9 \\
171.2\end{array}$ & 283.4 \\
\hline 8 & Oleic acid & 99.70 & 0.3 & - & $118 \pm 4$ & $526 \pm 2$ & $4.5 \pm 0.2$ & 66 & $\begin{array}{l}162.7 \\
170.3\end{array}$ & 285.9 \\
\hline 9 & Palm oil & 99.80 & 0.07 & 0.13 & $165 \pm 6$ & $682 \pm 9$ & $4.1 \pm 0.2$ & 66 & $\begin{array}{l}162.5 \\
171.1\end{array}$ & 277.6 \\
\hline 10 & Sunflower seed oil & 99.77 & 0.21 & 0.02 & $124 \pm 2$ & $479 \pm 4$ & $3.9 \pm 0.1$ & 70 & $\begin{array}{l}160.7 \\
169.6\end{array}$ & 267.6 \\
\hline 11 & Siberian oilseed oil & 99.71 & 0.25 & 0.04 & $149 \pm 9$ & $511 \pm 21$ & $3.4 \pm 0.2$ & 65 & $\begin{array}{l}160.5 \\
169.8\end{array}$ & 285.3 \\
\hline
\end{tabular}

"-" no such monomer in the PHA. 


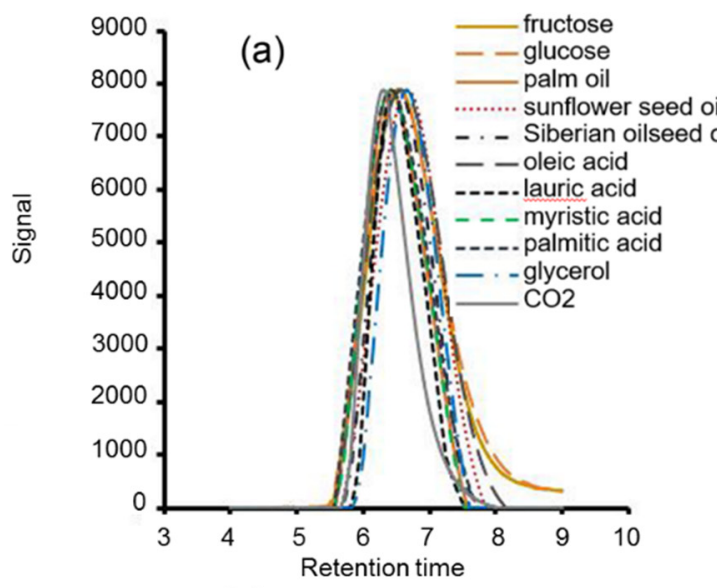

(c)

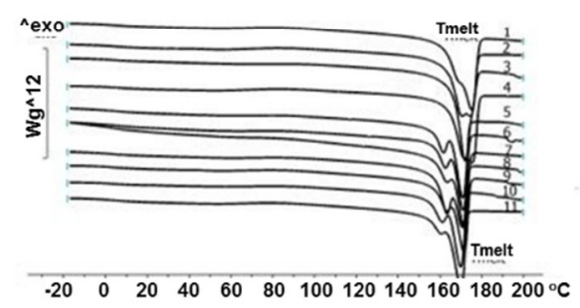

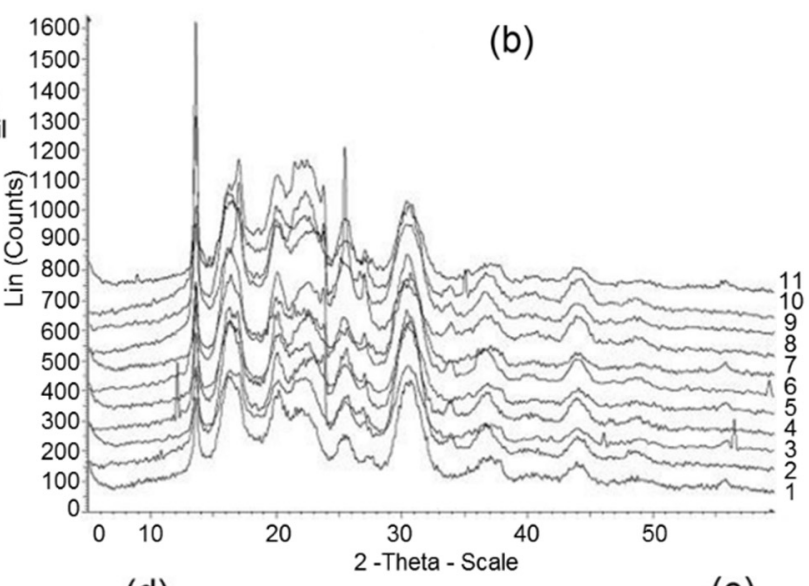

(d)

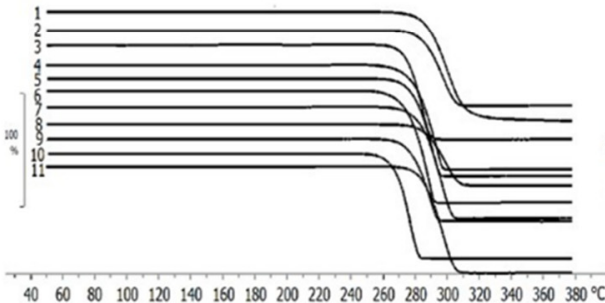

(e)

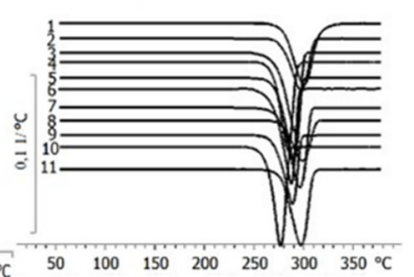

Figure 4. Physicochemical properties of PHAs synthesized by Cupriavidus necator IBP/SFU-1 from various carbon sources: (a) molecular weight distribution chromatogram, (b) the X-ray, (c) DSC, (d) TGA, (e) DTG (numbering of Samples 1-11 is the same as in Table 1).

A number of studies analyzed the properties of PHAs synthesized from glycerol, placing a special emphasis on molecular-weight properties because glycerol has been reported to function as a chain transfer (CT) reaction agent in PHA polymerization, resulting in the formation of low-molecular-weight PHA. In case of a CT reaction, the PHA chain number increases in inverse proportion to the PHA molecular weight [68]. However, some of the data on the $\mathrm{M}_{\mathrm{W}}$ of the polymers synthesized on glycerol are contradictory. There are studies reporting the reduction in the $\mathrm{M}_{\mathrm{W}}$ of $\mathrm{P}(3 \mathrm{HB})$ synthesized from glycerol [52,69], but other authors show rather high $\mathrm{M}_{\mathrm{w}}$ values of the polymer produced from glycerol, which reach 620 and $750 \mathrm{kDa}$ [70] and even 790-960 kDa [71]. The study of the properties of $\mathrm{P}(3 \mathrm{HB})$ synthesized by $C$. necator $\mathrm{B}-10646$ from glycerol demonstrated that the degree of purity of glycerol affected polymer molecular weight [39]. The polymer synthesized from purified and distilled glycerol had reduced $\mathrm{M}_{\mathrm{W}}-355$ and $420 \mathrm{kDa}$, but the $\mathrm{M}_{\mathrm{w}}$ of the polymer synthesized from crude glycerol was even lower, $304 \mathrm{kDa}$.

PHAs are semi-crystalline polymers, as crystallization does not occur in all regions of the material. The degree of crystallinity is the least studied aspect of PHAs. $\mathrm{P}(3 \mathrm{HB})$ has been generally recognized as a high-crystallinity polymer, in which the crystalline phase prevails over the amorphous phase, constituting from 65-67 to $80 \%$ [72].

The division of the polymer into crystallites and amorphous regions and determination of the degree of crystallinity are a first-approximation structural scheme, which describes the supramolecular structure. As the new data are obtained, this scheme is developed and improved. However, the data on the degree of crystallinity are widely used in scientific and technical research because this parameter directly determines the mechanical and physical properties of the polymer. The degree of crystallinity determines hardness, density, transparency, and diffusion. The higher the degree of crystallinity, the harder and stronger, but more brittle the product is.

In the present study, differences in the degrees of crystallinity of the PHAs synthesized from the carbon sources were not statistically significant. The $C_{x}$ values varied within a rather narrow range (65-72\%) regardless of the type of carbon source and the structure of the polymers (Table 1). 
The thermal properties of PHAs and their ability to crystallize in a native state are important parameters that determine the thermomechanical properties and melt processability of the polymer. The considerable difference between the melting point and the thermal degradation temperature is an important processing property of the polymer and owing to this property, the polymer can be processed into different products from melts, using generally accepted methods (solvent molding, extrusion, injection molding). $\mathrm{P}(3 \mathrm{HB})$ has a softening point at approximately $110{ }^{\circ} \mathrm{C}$ and crystallization temperature $\left(\mathrm{T}_{\mathrm{C}}\right)$ between 65 and $91^{\circ} \mathrm{C}$. No significant differences were revealed between the thermal properties of the PHAs synthesized from various carbon sources by the new strain C. necator IBP/SFU-1 (Table 1, Figure $4 \mathrm{c}-\mathrm{e})$. All PHA specimens had similar melting points $\left(\mathrm{T}_{\text {melt }}\right)$ and thermal degradation temperatures $\left(\mathrm{T}_{\mathrm{degr}}\right)$, with no statistically significant differences between them. $\mathrm{T}_{\text {melt }}$ values ranged between 160 and $172{ }^{\circ} \mathrm{C}$ and $\mathrm{T}_{\text {degr }}$ values between 267 and $285^{\circ} \mathrm{C}$. Previous studies showed that glycerol [39] and plant oils [64] did not affect the thermal properties of PHAs synthesized by C. necator B10646.

The present study demonstrated that PHA composition and properties were determined not only by the nutrient sources such as precursor substrates supplementing the main carbon source but also by the characteristics of the PHA producing strain and the type of the growth carbon source. C. necator IBP/SFU-1 was found to be capable of synthesizing short- and medium-chain-length PHAs even when cultivated in the medium that had not been supplemented with precursor substrates. Hence, the addition of precursors of different monomers to the culture medium can be expected to increase the fractions of $3 \mathrm{HV}$, $3 \mathrm{HHx}$, and, probably, other monomers in the PHA copolymers synthesized by this strain.

The review of the literature on physicochemical properties of PHAs revealed that the data differed substantially even when the specimens described in the studies were similar in their chemical composition. The authors of the study [73] noted that the data on the molecular weight of $\mathrm{P}(3 \mathrm{HB})$ reported by different researchers differed by one order of magnitude and the data on $\mathrm{P}(3 \mathrm{HB}) \mathrm{T}_{\text {melt }}$ varied between 162 and $197{ }^{\circ} \mathrm{C}$. The $\mathrm{T}_{\text {melt }}$ of $\mathrm{P}(3 \mathrm{HB}-\mathrm{co}-3 \mathrm{HV})$ copolymers with the same $3 \mathrm{HV}$ content ranged between 56 and $186{ }^{\circ} \mathrm{C}$ [10]. In the study [73], 3HV content of up to $20-22 \mathrm{~mol} \%$ did not influence the degree of crystallinity of $\mathrm{P}(3 \mathrm{HB}-\mathrm{co}-3 \mathrm{HV})$, whereas, in another study [74], the $\mathrm{C}_{\mathrm{x}}$ of the same copolymer was considerably reduced (to 5-9\%). The degree of crystallinity of the $\mathrm{P}(3 \mathrm{HB}-$ co-3HHx) copolymer containing 3HHx approximately $12-18 \mathrm{~mol} \%$ was $38-40 \%$ [74], but in the study [75], the same degree of crystallinity was determined for the copolymer with $3 \mathrm{HHx}$ content of only $1.5 \mathrm{~mol} \%$. The reason for such differences could be that the authors used different strains and carbon sources, collected polymer samples in different phases of the culture, used dissimilar methods of recovery and purification of polymers, etc. Thus, these factors should be taken into account in studies of the properties of PHAs and PHA-based products.

In addition, there are no definitive data showing how the basic properties of PHAs change depending on the composition of the medium and cell growth conditions and how these changes affect the properties of the PHA products. At the same time, some researchers reported that the chemical composition of PHA copolymers and the monomers constituting them influenced the surface microstructure and properties of polymer films [42,76] and their biological compatibility and blood cell response [77]. Replacement of glucose by plant oils containing glycerol not only resulted in changes of molecular weight and crystallinity of $\mathrm{P}(3 \mathrm{HB})$ synthesized by $\mathrm{C}$. necator $\mathrm{H} 16$ but also considerably affected the structure and properties of nonwoven membranes fabricated using electrospinning technique [78]. A similar effect of the type of carbon source on the properties of polymer films was described in [76]: comparison of the surface properties of the films prepared from $\mathrm{P}(3 \mathrm{HB})$ synthesized by C. necator B-10646 from glucose and glycerol revealed a considerable reduction in water contact angles of the surfaces of the films prepared from the polymer synthesized from glycerol.

Figures 5-7 show results of examining the films fabricated from PHAs synthesized 
from various carbon sources by C. necator IBP/SFU-1. SEM images illustrate differences in surface morphology (Figure 5).

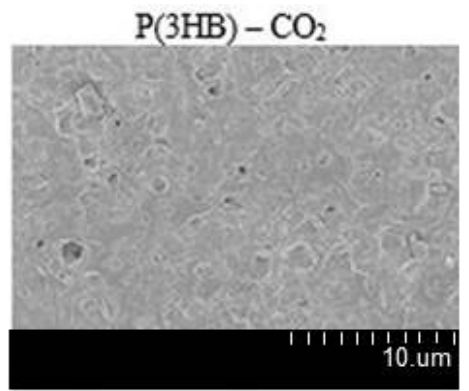

$\mathrm{P}(3 \mathrm{HB})$ - glycerol

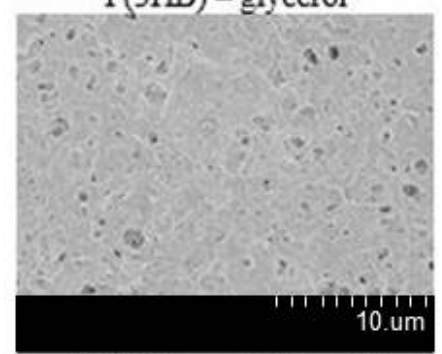

$\mathrm{P}(3 \mathrm{HB})$ - palmitic acid

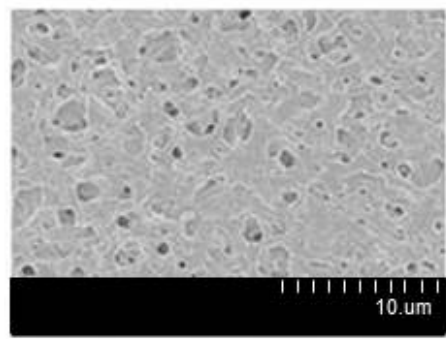

$\mathrm{P}(3 \mathrm{HB}-\mathrm{co}-3 \mathrm{HV}-3 \mathrm{HHx})-$ Siberian oilseed oil

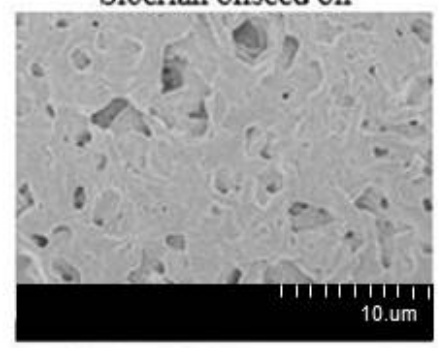

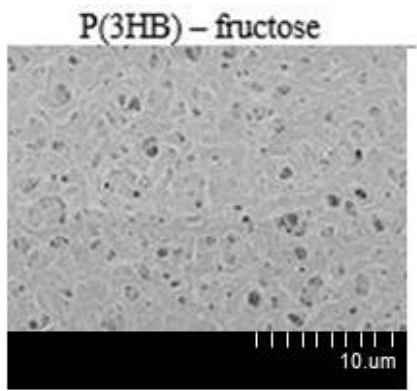
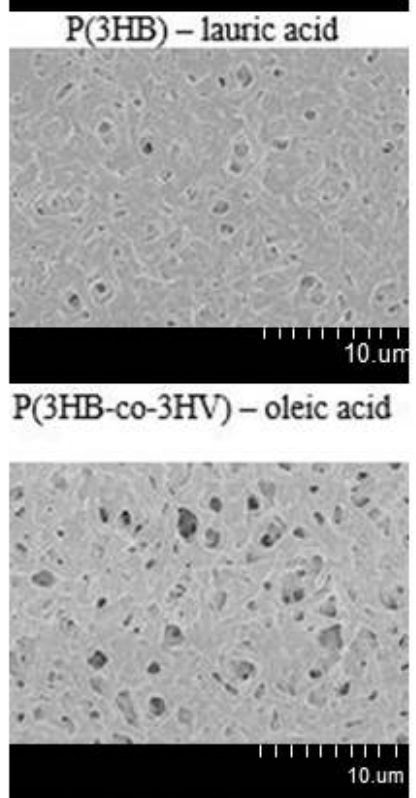

$\mathrm{P}(3 \mathrm{HB}-\mathrm{Co}-3 \mathrm{HV}-3 \mathrm{HHx})-$ sunflower seed oil

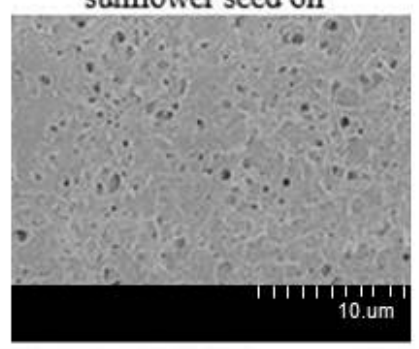

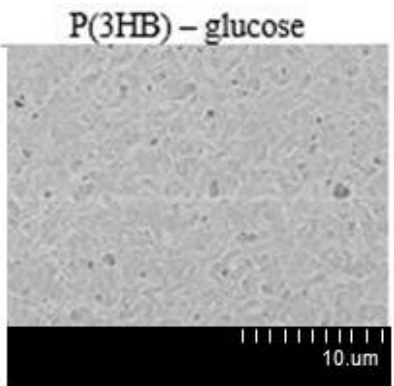

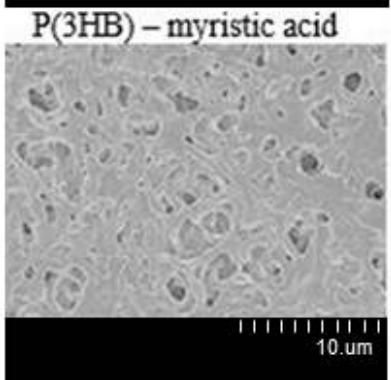

P(3HB-co-3HV-co-3HHx) - palm oil

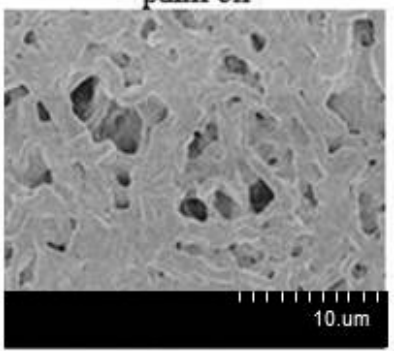

Figure 5. SEM images of the films fabricated from PHA synthesized from different carbon sources by Cupriavidus necator IBP/SFU-1.

The surfaces of the films fabricated from PHAs synthesized from various carbon substrates differed in the number and size of pores. That was most likely caused by differences in the kinetics of crystallization during the formation of the films, as they dry and the solvent evaporates.

Comparison of the porosity of films fabricated from one type of PHA, poly(3-hydro- xybutyrate), showed that the number of pores was similar for all seven samples (32.8-46.5/1000 $\mu^{2}$ ) while the pore sizes of these samples differed significantly. Thus, the average pore area was minimal, 1.9 and $2.4 \mu \mathrm{m}^{2}$, for the films fabricated from $\mathrm{P}(3 \mathrm{HB})$ synthesized from glucose and lauric acid, respectively. All copolymer films, except the films fabricated from the $\mathrm{P}(3 \mathrm{HB}-\mathrm{co}-3 \mathrm{HV}-\mathrm{co} 3 \mathrm{HHx})$ terpolymer synthesized from sunflower oil, had larger pore areas. The average pore area was $14.0,21.7$, and $24.7 \mu \mathrm{m}^{2}$, respectively, for films fabricated from the $\mathrm{P}(3 \mathrm{HB} / 3 \mathrm{HV})$ copolymer synthesized from oleic acid and films 
fabricated from $\mathrm{P}(3 \mathrm{HB}-3 \mathrm{HV}-\mathrm{co}-3 \mathrm{HHx})$ synthesized from palm oil and Siberian oilseed oil (data not shown).

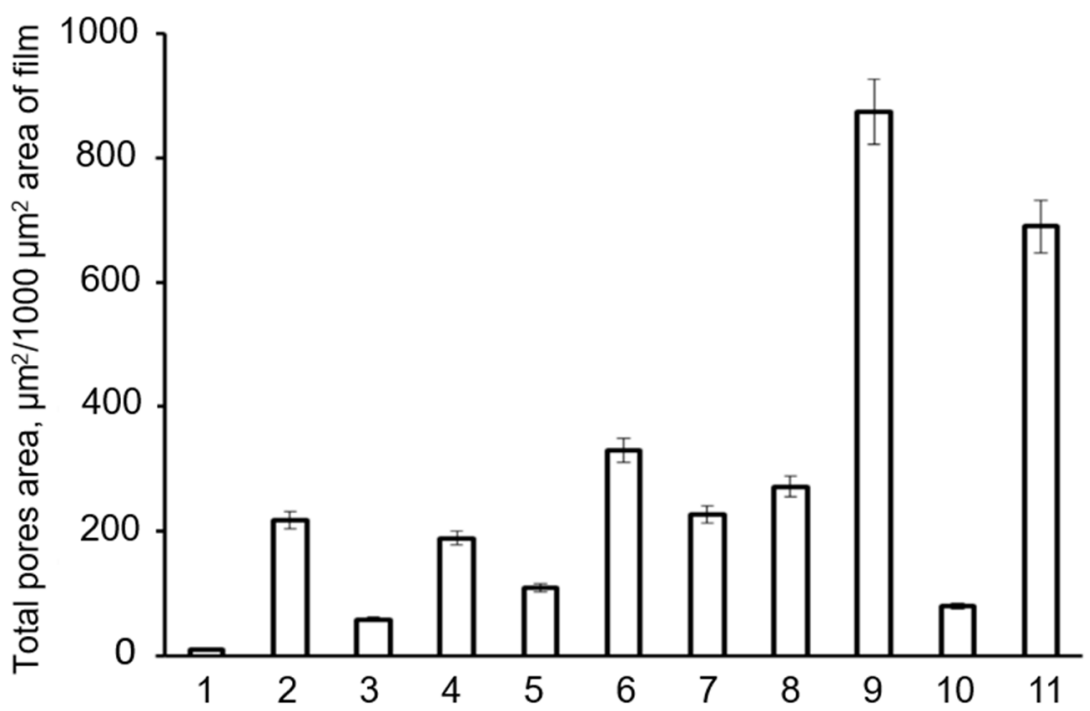

Figure 6. Total pores areas of films fabricated from PHAs synthesized from different carbon sources by Cupriavidus necator IBP/SFU-1 (numbering of Samples 1-11 is the same as in Table 1).

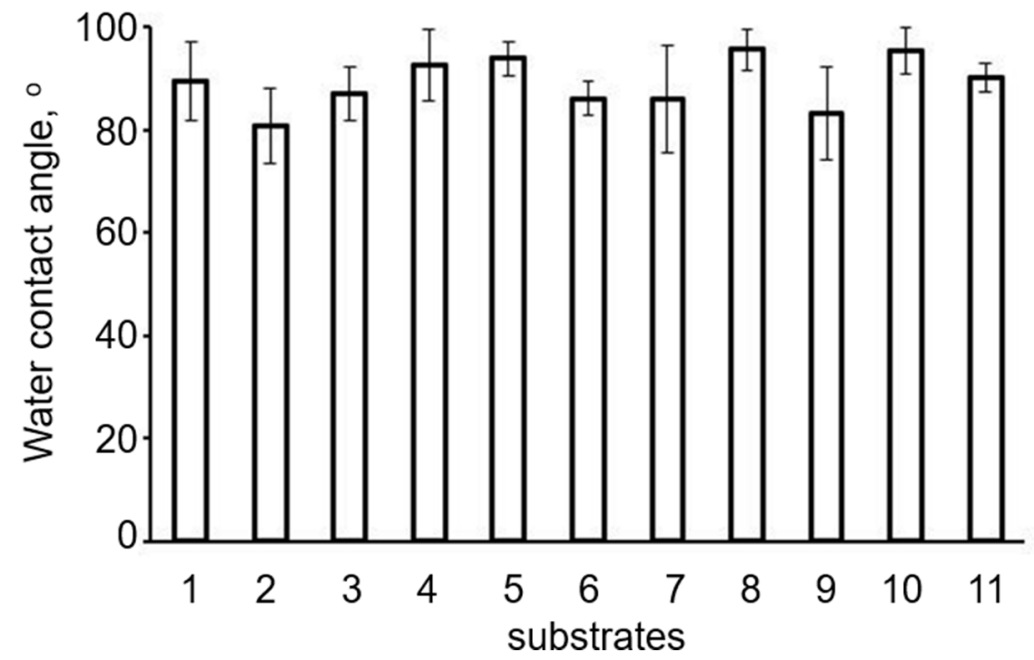

Figure 7. Water contact angles of the films prepared from PHAs synthesized from various carbon sources by Cupriavidus necator IBP/SFU-1 (numbering of Samples 1-11 is the same as in Table 1).

These differences are reflected in the integrated indicator, the total pore area (Figure 6).

The lowest values of the total pore area $\left(11 \mu \mathrm{m}^{2} / 1000 \mu \mathrm{m}^{2}\right.$ of the film surface area) were found for the films fabricated from $\mathrm{P}(3 \mathrm{HB})$ synthesized under autotrophic conditions. The total pore areas of 59 and $110 \mu \mathrm{m}^{2} / 1000 \mu \mathrm{m}^{2}$ were found for the films of $\mathrm{P}(3 \mathrm{HB})$ synthesized from glucose and lauric acid and for the film synthesized from sunflower oil $\left(80 \mu \mathrm{m}^{2} / 1000 \mu \mathrm{m}^{2}\right)$. The pore areas of the copolymer films were several times greater. The highest values $\left(690\right.$ and $874 \mu \mathrm{m}^{2} / 1000 \mu \mathrm{m}^{2}$ ) were found for the films fabricated from $\mathrm{P}(3 \mathrm{HB}-\mathrm{co}-3 \mathrm{HV}-3 \mathrm{HHx})$ synthesized from Siberian oilseed oil and palm oil. Thus, films with different porosities can be fabricated using PHAs synthesized from various C-substrates differing in their monomer compositions.

Thus, solution-cast films prepared from PHAs with different chemical compositions had surfaces differing in the number and size of pores. That could be associated with differences in the crystallization kinetics during solvent evaporation and formation of films from polymers with different degrees of crystallinity, which could affect the attachment and 
development of eukaryotic cells. The carbon source used to synthesize a PHA did not affect the water contact angle, which is an indirect indicator of surface hydrophilicity. The water contact angles of all films were similar (Figure 6). Films fabricated from $\mathrm{P}(3 \mathrm{HB})$ specimens synthesized from glucose, glycerol, and FAs showed almost equal water contact angles.

The decrease in the values of the water contact angle of the films (Figure 7) prepared from $\mathrm{P}(3 \mathrm{HB})$ synthesized from fructose and $\mathrm{P}(3 \mathrm{HB}-\mathrm{co}-3 \mathrm{HV}-\mathrm{co}-3 \mathrm{HHx})$ synthesized from palm oil to $80.8 \pm 7.33$ and $83.3 \pm 9.0^{\circ}$, respectively, was non-significant (at the confidence level $\alpha=0.05)$. Another study [76], though, demonstrated a significant decrease in the water contact angles of the films prepared from $\mathrm{P}(3 \mathrm{HB})$ specimens synthesized from glycerol compared to sugars. In that study, however, polymer synthesized from glycerol showed a considerably (50-55\%) decreased crystallinity. The increase in the values of the water contact angle of the films prepared from PHA copolymers synthesized from sunflower seed oil and oleic acid $\left(95.5-95.7^{\circ}\right)$ was not statistically significant either. Thus, none of the film types prepared from PHAs synthesized from various carbon sources by $C$. necator IBP/SFU-1 and having similar degrees of crystallinity and thermal properties showed any significant differences in the values of the water contact angle.

\section{Conclusions}

The bacterial strain isolated from the soil in the Krasnoyarsk Territory was studied as a PHA producer. The bacterial strain was identified as Cupriavidus necator IBP/SFU-1 based on the morphological, culture, and molecular-genetic characters. The strain was used as inoculum in suspension culture and batch-cultured to study cell growth and PHA synthesis from different carbon sources. The strain was found to be able to grow and synthesize PHAs under autotrophic conditions on $\mathrm{CO}_{2}$ mixed with $\mathrm{H}_{2}$ and $\mathrm{O}_{2}$ and showed a broad organotrophic potential towards different carbon sources: sugars, glycerol, fatty acids, and various plant oils. The highest cell concentrations and PHA content were produced in the cultures with palm oil and oleic acid used as carbon sources. Cultivation on the widely available palm oil resulted in cell concentration and intracellular polymer content reaching 8.2 and $6.56 \mathrm{~g} / \mathrm{L}$, respectively, and the highest productivity $(2.73 \mathrm{~g} / \mathrm{L} \mathrm{d})$ and high $(80 \%)$ polymer content. On oleic acid, cell concentration, and intracellular polymer content were 8.0 and $6.27 \mathrm{~g} / \mathrm{L}$, corresponding to biomass productivity of $2.67 \mathrm{~g} / \mathrm{L} \cdot \mathrm{d}$ and polymer content of $78.4 \%$. Results obtained on sugars (fructose and glucose) did not differ considerably, cell concentrations were 7.0 and $7.7 \mathrm{~g} / \mathrm{L}$ and intracellular polymer contents were 5.68 and $6.33 \mathrm{~g} / \mathrm{L}$, respectively, with productivities of 2.33 and $2.57 \mathrm{~g} / \mathrm{L} \mathrm{d}$ and polymer content of 81.1 and $84.3 \%$. Cultivation on purified glycerol produced somewhat lower results, cell concentration and intracellular polymer content were 5.0 and $3.26 \mathrm{~g} / \mathrm{L}$ and productivity reached $1.67 \mathrm{~g} / \mathrm{L} \mathrm{d}$ and $65.1 \%$. Production parameters of the strain grown on other substrates (saturated fatty acids and sunflower seed oil and Siberian oilseed oil) were even lower.

The study showed that PHA chemical composition and properties were determined by the carbon source used. When grown on oleic acid, the strain synthesized the $\mathrm{P}\left(3 \mathrm{HB}-\mathrm{co}^{-}\right.$ $3 \mathrm{HV})$ copolymer; on Siberian oilseed oil, sunflower seed oil, and palm oil-the $\mathrm{P}(3 \mathrm{HB}-\mathrm{co}-$ $3 \mathrm{HV}-\mathrm{co}-3 \mathrm{HHx})$ terpolymer, and on the other substrates-the $\mathrm{P}(3 \mathrm{HB})$ homopolymer. In all copolymer types, 3-hydroxybutyrate constituted over 99 mol.\%, and 3-hydroxyvalerate and the medium-chain-length 3-hydroxyhexanoate were present in minor amounts. The type of the carbon source mainly influenced molecular-weight properties of PHAs: molecular weight and polydispersity. $\mathrm{P}(3 \mathrm{HB})$ synthesized under autotrophic growth conditions, from $\mathrm{CO}_{2}$, had the highest number-average $(290 \pm 15 \mathrm{kDa})$ and weight-average $(850 \pm 25 \mathrm{kDa})$ molecular weights and the lowest polydispersity (2.9 \pm 0.2$)$. All polymers synthesized from organic carbon sources, regardless of PHA composition, showed increased polydispersity and reduced molecular weight; $\mathrm{P}(3 \mathrm{HB})$ synthesized from glycerol had the lowest $\mathrm{M}_{\mathrm{n}}$ and $\mathrm{M}_{\mathrm{w}}$. All PHAs, regardless of the type of the carbon source used, had similar degrees of crystallinity, with the ordered phase prevailing in the polymers (64-72\%); no effect of the carbon source on thermal properties of the PHAs was found either. The type of the 
carbon source determined not only PHA composition and properties but also the surface microstructure and porosity of polymer films. Results obtained in this study show that the strain Cupriavidus necator IBP/SFU-1 has a broad organotrophic potential and is capable of synthesizing PHAs from various carbon substrates, producing high cell concentrations and intracellular contents of polymers, including short- and medium-chain-length copolymers. Thus, this strain can be regarded as a promising $\mathrm{P}(3 \mathrm{HB})$ producer from palm oil, oleic acid, and sugars (fructose and glucose) and as a producer of $\mathrm{P}(3 \mathrm{HB}-\mathrm{co}-3 \mathrm{HV})$ from oleic acid and $\mathrm{P}(3 \mathrm{HB}-\mathrm{co}-3 \mathrm{HV}-\mathrm{co}-3 \mathrm{HHx})$ from palm oil.

Author Contributions: Isolation of the strain, PHA synthesis, N.O.Z.; film production, K.Y.S.; PHA properties, A.D.V. and E.G.K.; SEM of the films, I.V.N.; surface properties of the films E.I.S.; conceptualization, methodology, analysis, T.G.V. All authors have read and agreed to the published version of the manuscript.

Funding: This work was financially supported by Project "Agropreparations of the new generation: a strategy of construction and realization" (Agreement No 075-15-2021-626) in accordance with Resolution No 220 of the Government of the Russian Federation of 9 April 2010, "On measures designed to attract leading scientists to the Russian institutions of higher learning" (strain isolation, polymer synthesis and investigation), and by the State Assignment of the Ministry of Science and Higher Education of the Russian Federation No. FSRZ-2020-0006 (study of film properties).

Institutional Review Board Statement: Not applicable.

Informed Consent Statement: Not applicable.

Data Availability Statement: Not applicable.

Acknowledgments: The authors would like to express their special thanks to the Krasnoyarsk Regional Center of Research Equipment of the Federal Research Center "Krasnoyarsk Science Center SB RAS" for providing equipment to ensure the accomplishment of this project.

Conflicts of Interest: The authors declare that they have no conflict of interest in the publication of this article. The authors have no conflicts of interest to report in this work. The manuscript was written through the contributions of all authors. All authors have approved the final version of the manuscript.

\section{References}

1. Lebreton, L.; Andrady, A. Future scenarios of global plastic waste generation and disposal. Palgrave Commun. 2019, 5, 6. [CrossRef]

2. Quecholac-Piña, X.; Hernández-Berriel, M.; Mañón-Salas, M.; Espinosa-Valdemar, R.M.; Vázquez-Morillas, A. Degradation of plastics under anaerobic conditions: A short review. Polymers 2020, 12, 109. [CrossRef]

3. Sohn, Y.J.; Kim, H.T.; Baritugo, K.A.; Jo, S.Y.; Song, N.M.; Park, S.Y.; Park, S.K.; Pyo, J.; Cha, H.G.; Kim, H.; et al. Recent advances in sustainable plastic upcycling and biopolymers. Biotechnol. J. 2020, 15, 1900489. [CrossRef]

4. Geyer, R.; Jambeck, J.R.; Law, K.L. Production, use, and fate of all plastics ever made. Law Sci. Adv. 2017, 3, 170-782. [CrossRef] [PubMed]

5. Katyal, D.; Kong, E.; Villanueva, J. Microplastics in the environment: Impact on human health and future mitigation strategies. Environ. Health Rev. 2020, 63, 27-31. [CrossRef]

6. Urbanek, A.K.; Rymowicz, W.; Mirończuk, A.M. Degradation of plastics and plastic-degrading bacteria in cold marine habitats. Appl. Microbiol. Biotechnol. 2018, 102, 7669-7678. [CrossRef]

7. Mannina, G.; Presti, D.; Montiel-Jarillo, G.; Suárez-Ojeda, M.E. Bioplastic recovery from wastewater: A new protocol for polyhydroxyalkanoates (PHA) extraction from mixed microbial cultures. Bioresour. Technol. 2019, 282, 361-369. [CrossRef]

8. Huang, L.; Chen, Z.; Wen, Q.; Ji, Y.; Wu, Z.; Lee, D.J. Toward flexible regulation of polyhydroxyalkanoate composition based on substrate feeding strategy: Insights into microbial community and metabolic features. Bioresour. Technol. 2020, 296, 122369. [CrossRef] [PubMed]

9. Volova, T.G.; Shishatskaya, E.I.; Sinskey, A.J. Degradable Polymers: Production, Properties, Applications; Nova Science Pub Inc.: Hauppauge, NY, USA, 2013; 380p.

10. Laycock, B.; Halley, P.; Pratt, S.; Werker, A.; Lant, P. The chemomechanical properties of microbial polyhydroxyalkanoate. Prog. Polym. Sci. 2013, 38, 536-583. [CrossRef]

11. Volova, T.G.; Zhila, N.O.; Shishatskaya, E.I.; Mironov, P.V.; Vasil'ev, A.D.; Sukovatyi, A.G.; Sinskey, A.J. The physicochemical properties of polyhydroxyalkanoates with different chemical structures. Polym. Sci. Ser. A 2013, 55, 427-437. [CrossRef]

12. Zheng, J.; Sue, S. Strategies to reduce the global carbon foot print of plastics. Nat. Clim. Chang. 2019, 9, 374-378. [CrossRef] 
13. Chen, G.Q.; Jiang, X.R.; Guo, Y. Synthetic biology of microbes synthesizing polyhydroxyalkanoates (PHA). Synth. Syst. Biotechnol. 2016, 1, 236-242. [CrossRef] [PubMed]

14. Koller, M.; Mukherjee, A. Polyhydroxyalkanoates-Linking properties, applications, and end-of-life options. Chem. Biochem. Eng. Q. 2020, 34, 115-129. [CrossRef]

15. Tarrahi, R.; Fathi, Z.; Özgür, M.; Seydibeyoğlu, K.; Doustkhah, E.; Khataee, A. Polyhydroxyalkanoates (PHA): From production to nanoarchitecture. Int. J. Biol. Macromol. 2020, 146, 596-619. [CrossRef]

16. Chen, G.-Q. Plastics completely synthesized by bacteria: Polyhydroxyalkanoates. In Plastics from Bacteria: Natural Functions and Applications; Chen, G.-Q., Ed.; Springer: Berlin/Heidelberg, Germany, 2010; pp. 17-37.

17. Sudesh, K.; Abe, H. Practical Guide to Microbial Polyhydroxyalkanoates; A Smithers Group Company: Shropshire, UK, 2010; 158p.

18. Volova, T.G.; Vinnik, Y.S.; Shishatskaya, E.I.; Markelova, N.M.; Zaikov, G.E. Natural-Based Polymers for Biomedical Applications; Apple Academic Press: New York, NY, USA, 2017; 460p.

19. Volova, T.G.; Shishatskaya, E.I.; Prudnikova, S.V.; Zhila, N.O.; Boyandin, A.N. New Generation Formulations of Agrochemicals: Current Trends and Future Priorities; Apple Academic Press: Boca Raton, FL, USA, 2020; 286p.

20. Ansari, S.; Sami, N.; Yasin, D.; Ahmad, N.; Fatma, T. Biomedical applications of environmental friendly poly-hydroxyalkanoates. Int. J. Biol. Macromol. 2021, 183, 549-563. [CrossRef]

21. Hinchliffe, J.D.; Madappura, A.P.; Mohamed, S.M.D.S.; Roy, I. Review: Biomedical applications of bacteria-derived polymers. Polymers. 2021, 13, 1081. [CrossRef]

22. Koller, M. The Handbook of Polyhydroxyalkanoates: Postsynthetic Treatment, Processing and Application; CRC Press: Boca Raton, FL, USA, 2020; Volume 3.

23. Vandamme, P.; Coenye, T. Taxonomy of the genus Cupriavidus: A tale of lost and found. Int. J. Syst. Evol. Microbiol. 2004, 54, 2285-2289. [CrossRef]

24. Schlegel, H.G.; Kaltwasser, H.; Gottschalk, G. Ein Submersverfahren zur kultur wasserstoffoxydierender bakterien: Wachstumphysiologische untersuchungen. Arch. Mikrobiol. 1961, 38, 209-222. [CrossRef]

25. Zavarzin, G.A. Vodorodnyye Bakterii i Karboksidobakterii (Hydrogen Bacteria and Carboxydobacteria); Nauka: Moscow, Russia, 1978; 203p.

26. Tanaka, K.; Ishizaki, A.; Kanamaru, T.; Kawano, T. Production of poly(D-3-hydroxybutyrate) from $\mathrm{CO}_{2}, \mathrm{H}_{2}$ and $\mathrm{O}_{2}$ by high cell density autotrophic cultivation of Alcaligenes eutrophus. Biotechnol. Bioeng. 1995, 45, 268-275. [CrossRef] [PubMed]

27. Tanaka, K.; Miyawaki, K.; Yamaguchi, A.; Khosravi-Darani, K.; Matsusaki, H. Cell growth and $\mathrm{P}(3 \mathrm{HB})$ accumulation from $\mathrm{CO}_{2}$ of a carbon monoxide-tolerant hydrogen-oxidizing bacterium, Ideonella sp. O-1. Appl. Microbiol. Biotechnol. 2011, 92, 1161-1169. [CrossRef] [PubMed]

28. Volova, T.G. Hydrogen-Based Biosynthesis; Nova Science Pub Inc.: Hauppauge, NY, USA, 2009; 287p.

29. Green, P.R.; Kemper, J.; Schechtman, L.; Guo, L.; Satkowski, M.; Fiedler, S.; Steinbüchel, A.; Rehm, B.H.A. Formation of short chain length/medium length polyhydroxyalkanoate copolymers by fatty acid b-oxidation inhibited Ralstonia eutropha. Biomacromolecules 2002, 3, 208-213. [CrossRef]

30. Chanprateep, S.; Kulpreecha, S. Production and characterization of biodegradable terpolymer poly(3-hydroxybutyrate-co-3hydroxyvalerate-co-4-hydroxybutyrate) by Alcaligenes sp. A-04. J. Biosci. Bioeng. 2006, 101, 51-56. [CrossRef] [PubMed]

31. Cavalheiro, J.M.B.T.; Raposo, R.S.; de Almeida, M.C.M.D.; Cesario, M.T.; Sevrin, C.; Grandfils, C.; da Fonseca, M.M.R. Effect of cultivation parameters on the production of poly(3-hydroxybutyrate-co-4-hydroxybutyrate) and poly(3-hydroxybutyrate4-hydroxybutyrate-3-hydroxyvalerate) by Cupriavidus necator using waste glycerol. Bioresour. Technol. 2012, 111, $391-397$. [CrossRef]

32. Chen, G.Q.; Chen, X.Y.; Wu, F.Q.; Chen, J.C. Polyhydroxyalkanoates (PHA) toward cost competitiveness and functionality. Adv. Ind. Eng. Polym. Res. 2020, 3, 1-7. [CrossRef]

33. Nandakumar, A.; Chuah, J.A.; Sudesh, K. Bioplastics: A boon or bane? Renew. Sustain. Energy Rev. 2021, 147, 111237. [CrossRef]

34. Sutton, D.; Fothergill, A.; Rinaldi, M. Guide to Pathogenic and Opportunistic Fungi; Trans. from English; Mir: Moscow, Russia, 2001; 486p.

35. Vos, P.D.; Garrity, G.M.; Jones, D.; Krieg, N.R.; Ludwig, W.; Rainey, F.A.; Schleifer, K.H.; Whitman, W.B. Bergey's Manual of Systematic Bacteriology, 2nd ed.; Volume 3: The Firmicutes; Springer: New York, NY, USA, 2009; 1450p.

36. Saitou, N.; Nei, M. The neighbor-joining method: A new method for reconstructing phylogenetic trees. Mol. Biol. Evol. 1987, 4, 406-425. [CrossRef] [PubMed]

37. Tamura, K.; Nei, M.; Kumar, S. Prospects for inferring very large phylogenies by using the neighbor-joining method. Proc. Natl. Acad. Sci. USA 2004, 101, 11030-11035. [CrossRef] [PubMed]

38. Kumar, S.; Stecher, G.; Li, M.; Knyaz, C.; Tamura, K. MEGA X: Molecular evolutionary genetics analysis across computing platforms. Mol. Biol. Evol. 2018, 35, 1547-1549. [CrossRef]

39. Volova, T.; Demidenko, A.; Kiselev, E.; Baranovskii, S.; Shishatskaya, E.; Zhila, N. Polyhydroxyalkanoate synthesis based on glycerol and implementation of the process under conditions of pilot production. Appl. Microbiol. Biotechnol. 2019, 103, $225-237$. [CrossRef]

40. Braunegg, G.; Sonnleitner, B.; Lafferty, R.M. A rapid gas chromatographic method for the determination of poly- $\beta$-hydroxybutyric acid in microbial biomass. Eur. J. Appl. Microbiol. Biotechnol. 1978, 6, 29-37. [CrossRef]

41. Kiselev, E.G.; Vasiliev, A.D.; Volova, T.G. Synthesis and characteristics of multicomponent PHA. J. Sib. Fed. Univ. Biol. 2021, 14, 97-113. [CrossRef] 
42. Volova, T.; Kiselev, E.; Nemtsev, I.; Lukyanenko, A.; Sukovatyi, A.; Kuzmin, A.; Ryltseva, G.; Shishatskaya, E. Properties of degradable PHAs with different monomer compositions. Int. J. Biol. Macromol. 2021, 182, 98-114. [CrossRef] [PubMed]

43. Zhila, N.O.; Kalacheva, G.S.; Volova, T.G. Fatty acid composition and polyhydroxyalkanoates production by Cupriavidus eutrophus B-10646 cells grown on different carbon sources. Process Biochem. 2015, 50, 69-78. [CrossRef]

44. Madison, L.L.; Huisman, G.W. Metabolic engineering of poly(3-hydroxyalkanoates): From DNA to plastic. Microbiol. Mol. Biol. Rev. 1999, 63, 21-53. [CrossRef]

45. Volova, T.G.; Trusova, M.Y.; Kalacheva, G.S.; Kozhevnicov, I.V. Physiological-biochemical properties and the ability to synthesize polyhydroxyalkanoates of the glucose-utilizing strain of the hydrogen bacterium Ralstonia eutropha B8562. Appl. Microbiol. Biotechnol. 2006, 73, 429-433. [CrossRef]

46. Holmes, P.A. Applications of PHB-A microbially produced biodegradable thermoplastic. Phys. Technol. 1985, 16, 32-36. [CrossRef]

47. Kim, B.C.; Lee, S.Y.; Chang, Y.K.; Chang, Y.K.; Woo, S.I. Production of poly(3-hydroxybutyric-co-3-polyhydroxyvaleric acid) by fed-batch culture of Alcaligenes eutrophus with substrate control using on-line glucose analyze. Enzym. Microb. Technol. 1994, 16, 556-561. [CrossRef]

48. Ryu, H.W.; Cho, K.S.; Kim, B.S.; Chang, H.N.; Shim, H.J. Mass production of poly(3-hydroxybutyrate) in fed-batch culture of Ralstonia eutropha with nitrogen and phosphate limitation. J. Microbiol. Biotechnol. 1999, 9, 751-756.

49. Fernández-Dacosta, C.; Posada, J.A.; Kleerebezem, R.; Cuellar, M.C.; Ramirez, A. Microbial community-based polyhydroxyalkanoates (PHAs) production from wastewater: Techno-economic analysis and ex-ante environmental assessment. Bioresour. Technol. 2015, 185, 368-377. [CrossRef]

50. Rodríguez-Contreras, A.; Koller, M.; de Sousa Dias, M.M.; Calafell-Monfort, M.; Braunegg, G.; Marqués-Calvo, M.S. Influence of glycerol on poly(3-hydroxybutyrate) production by Cupriavidus necator and Burkholderia sacchari. Biochem. Eng. J. 2015, 94, 50-57. [CrossRef]

51. Campos, M.I.; Figueiredo, T.V.B.; Sousa, L.S.; Druzian, J.I. The influence of crude glycerin and nitrogen concentrations on the production of PHA by Cupriavidus necator using a response surface methodology and its characterizations. Ind. Crop. Prod. 2014, 52, 338-346. [CrossRef]

52. Ashby, R.D.; Solaiman, D.K.Y.; Foglia, T.A. Synthesis of short-/medium-chain-length poly(hydroxyalkanoate) blends by mixed culture fermentation of glycerol. Biomacromolecules 2005, 6, 2106-2112. [CrossRef]

53. Kumar, P.; Jun, H.B.; Kim, B.S. Co-production of polyhydroxyalkanoates and carotenoids through bioconversion of glycerol by Paracoccus sp. strain. Int. J. Biol. Macromol. 2018, 107, 2552-2558. [CrossRef] [PubMed]

54. Budde, C.F.; Riedel, S.L.; Hübner, F.; Risch, S.; Popović, M.K.; Rha, C.; Sinskey, A.J. Growth and polyhydroxybutyrate production by Ralstonia eutropha in emulsified plant oil medium. Appl. Microbiol. Biotechnol. 2011, 89, 1611-1619. [CrossRef] [PubMed]

55. Budde, C.F.; Riedel, S.L.; Willis, L.B.; Rha, C.; Sinskey, A.J. Production of poly(3-hydroxybutyrate-co-3-hydroxyhexanoate) from plant oil by engineered Ralstonia eutropha strains. Appl. Environ. Microbiol. 2011, 77, 2847-2854. [CrossRef]

56. Riedel, S.L.; Bader, J.; Brigham, C.J.; Budde, C.F.; Yusof, Z.A.M.; Rha, C.; Sinskey, A.J. Production of poly(3-hydroxybutyrateco-3-hydroxyhexanoate) by Ralstonia eutropha in high cell density palm oil fermentations. Biotechnol. Bioeng. 2012, 109, 74-83. [CrossRef] [PubMed]

57. Sato, S.; Maruyama, H.; Fujiki, T.; Matsumoto, K. Regulation of 3-hydroxyhexanoate composition in PHBH synthesized by recombinant Cupriavidus necator H16 from plant oil by using butyrate as co-substrate. J. Biosci. Bioeng. 2015, 120, $246-251$. [CrossRef]

58. Zainab-L, I.; Uyama, H.; Li, C.; Shen, Y.; Sudesh, K. Production of polyhydroxyalkanoates from underutilized plant oil by Cupriavidus necator. CLEAN-Soil Air Water 2018, 46, 1700542. [CrossRef]

59. Pérez-Arauz, A.O.; Aguilar-Rabiela, A.E.; Vargas-Torres, A.; Rodríguez-Hernández, A.I.; Chavarría-Hernández, N.; VergaraPorras, B.; López-Cuellar, M.R. Production and characterization of biodegradable films of a novel polyhydroxyalkanoate (PHA) synthesized from peanut oil. Food Packag. Shelf Life 2019, 20, 100297. [CrossRef]

60. Zhila, N.O.; Kalacheva, G.S.; Kiselev, E.G.; Volova, T.G. Synthesis of polyhydroxyalkanoates from oleic acid by Cupriavidus necator B-10646. J. Sib. Fed. Univ. Biol. 2020, 13, 208-217. [CrossRef]

61. Srivastava, S.K.; Tripathi, A.D. Effect of saturated and unsaturated fatty acid supplementation on bio-plastic production under submerged fermentation. 3 Biotech 2013, 3, 389-397. [CrossRef]

62. Iqbal, N.M.; Amirul, A.A. Synthesis of $\mathrm{P}(3 \mathrm{HB}-\mathrm{co}-4 \mathrm{HB})$ copolymer with target-specific $4 \mathrm{HB}$ molar fractions using combinations of carbon substrates. J. Chem. Technol. Biotechnol. 2013, 89, 407-418. [CrossRef]

63. Ramachandran, H.; Amirul, A.A. Yellow-pigmented Cupriavidus sp., a novel bacterium capable of utilizing glycerine pitch for the sustainable production of P(3HB-co-4HB). J. Chem. Technol. Biotechnol. 2013, 88, 1030-1038. [CrossRef]

64. Volova, T.; Sapozhnikova, K.; Zhila, N. Cupriavidus necator B-10646 growth and polyhydroxyalkanoates production on different plant oils. Int. J. Biol. Macromol. 2020, 164, 121-130. [CrossRef] [PubMed]

65. Lee, W.H.; Loo, C.Y.; Nomura, C.T.; Sudesh, K. Biosynthesis of polyhydroxyalkanoate copolymers from mixtures of plant oils and 3-hydroxyvalerate precursors. Bioresour. Technol. 2008, 99, 6844-6851. [CrossRef] [PubMed]

66. Obruca, S.; Marova, I.; Snajdar, O.; Mravcova, L.; Svoboda, Z. Production of poly(3-hydroxybutyrate-co-3-hydroxyvalerate) by Cupriavidus necator from waste rapeseed oil using propanol as a precursor of 3-hydroxyvalerate. Biotechnol. Lett. 2010, 32, 1925-1932. [CrossRef] 
67. Madden, L.A.; Anderson, A.J.; Shah, D.T.; Asrar, J. Chain termination in polyhydroxyalkanoate synthesis: Involvement of exogenous hydroxy-compounds as chain transfer agents. Int. J. Biol. Macromol. 1999, 25, 43-53. [CrossRef]

68. Tomozawa, S.; Saito, Y.; Nakamura, Y.; Abe, H.; Tsuge, T. Chain transfer reaction catalyzed by various PHA synthases with poly(ethylene glycol) as an exogenous chain transfer agent. Appl. Microbiol. Biotechnol. 2010, 87, 1427-1435. [CrossRef]

69. Ashby, R.D.; Solaiman, D.K.Y.; Strahan, G.D. Efficient utilization of crude glycerol as fermentation substrate in the synthesis of poly(3-hydroxybutyrate) biopolymers. J. Am. Oil Chem. Soc. 2011, 88, 949-959. [CrossRef]

70. Mothes, G.; Schnorpfeil, C.; Ackermann, J.U. Production of PHB from crude glycerol. Eng. Life Sci. 2007, 7, 475-479. [CrossRef]

71. Cavalheiro, J.M.B.T.; de Almeida, M.C.M.D.; Grandfils, C.; da Fonseca, M.M.R. Poly(3-hydroxybutyrate) production by Cupriavidus necator using waste glycerol. Process Biochem. 2009, 44, 509-515. [CrossRef]

72. Sudesh, K.; Abe, H.; Doi, Y. Synthesis, structure and properties of polyhydroxyalkanoates: Biological polyesters. Prog. Polym. Sci. 2000, 25, 1503-1555. [CrossRef]

73. Noda, I.; Green, P.R.; Satkowski, M.M.; Schechtman, L.A. Preparation and properties of novel class of polyhydroxyalkanoate copolymers. Biomacromolecules 2005, 6, 580-586. [CrossRef]

74. Dai, Y.; Yuan, Z.; Jack, K.; Keller, J. Production of targeted poly(3-hydroxyalkanoates) copolymers by glycogen accumulating organisms using acetate as sole carbon source. J. Biotechnol. 2007, 129, 489-497. [CrossRef]

75. Fukui, T.; Abe, H.; Doi, Y. Engineering of Ralstonia eutropha for production of poly(3-hydroxybutyrate-co-3-hydroxyhexanoate) from fructose and solid-state properties of the copolymer. Biomacromolecules 2002, 3, 618-624. [CrossRef] [PubMed]

76. Shishatskaya, E.; Nemtsev, I.; Lukyanenko, A.; Vasiliev, A.; Kiselev, E.; Sukovatyi, A.; Volova, T. Polymer films of poly-3hydroxybutyrate synthesized by Cupriavidus necator from different carbon sources. J. Polym. Environ. 2021, 29, 837-850. [CrossRef]

77. Shishatskaya, E.I.; Menzyanova, N.G.; Shumilova, A.A. The effect of the chemical composition and structure of polymer films made from resorbable polyhydroxyalkanoates on blood cell response. Int. J. Biol. Macromol. 2019, 141, 765-774. [CrossRef]

78. Irorere, V.U.; Bagheriasl, S.; Blevins, M.; Kwiecien, I.; Stamboulis, A.; Radecka, I. Electrospun fibres of polyhydroxybutyrate synthesized by Ralstonia eutropha from different carbon sources. Int. J. Polym. Sci. 2014, 2014, 705359. [CrossRef] 